\title{
UCRL-BOOK-203536
}

LAW RENCE LIVERMORE N A T IO N A L LABORATORY

\section{Solid Nitrogen at Extreme Conditions of High Pressure and Temperature}

A. Goncharov, E. Gregoryanz

April 15, 2004

Chemistry at Extreme Conditions 
This document was prepared as an account of work sponsored by an agency of the United States Government. Neither the United States Government nor the University of California nor any of their employees, makes any warranty, express or implied, or assumes any legal liability or responsibility for the accuracy, completeness, or usefulness of any information, apparatus, product, or process disclosed, or represents that its use would not infringe privately owned rights. Reference herein to any specific commercial product, process, or service by trade name, trademark, manufacturer, or otherwise, does not necessarily constitute or imply its endorsement, recommendation, or favoring by the United States Government or the University of California. The views and opinions of authors expressed herein do not necessarily state or reflect those of the United States Government or the University of California, and shall not be used for advertising or product endorsement purposes. 


\title{
Solid nitrogen at extreme conditions of high pressure and temperature
}

\author{
Alexander Goncharov ${ }^{a, b}$ and Eugene Gregoryanz ${ }^{a}$ \\ ${ }^{\mathrm{a}}$ Geophysical Laboratory, Carnegie Institution of Washington, 5251 Broad Branch Road, \\ Washington, D.C., 20015, USA. \\ ${ }^{\mathrm{b}}$ Lawrence Livermore National Laboratory, University of California, 7000 East Avenue, \\ Livermore, CA 94551, USA.
}

\begin{abstract}
We review the phase diagram of nitrogen in a wide pressure and temperature range. Recent optical and x-ray diffraction studies at pressures up to $300 \mathrm{GPa}$ and temperatures in excess of $1000 \mathrm{~K}$ have provided a wealth of information on the transformation of molecular nitrogen to a nonmolecular (polymeric) semiconducting and two new molecular phases. These newly found phases have very large stability (metastability) range. Moreover, two new molecular phases have considerably different orientational order from the previously known phases. In the iota phase (unlike most of other known molecular phases), $\mathrm{N}_{2}$ molecules are orientationally equivalent. The nitrogen molecules in the theta phase might be associated into larger aggregates, which is in line with theoretical predictions on polyatomic nitrogen.
\end{abstract}

\section{INTRODUCTION}

The evolution of molecular solids under pressure constitutes an important problem in condensed-matter physics [1]. Under compression, delocalization of electronic shells and eventual molecular dissociation is expected, leading to the formation of a framework or close-packed structures. However, this process may not necessarily be simple and direct, because of large barriers of transformation between states with different types of bonding and molecular structures with various types of orientational order, including possible associated and charge-transfer intermediate states. Nitrogen is an archetypal homonuclear diatomic molecule with a very strong triple bond. It was expected to undergo the transition to a network structure related to a destabilization of its triple bond in the pressure range accessible by modern experimental techniques. This prediction is based on the results of shock-wave experiments, which provided evidence for the transition to a nonmolecular phase in the liquid phase at $30 \mathrm{GPa}$ and $6000 \mathrm{~K}$ [2], as well as theoretical calculations for the solid [3-5]. The latter predictions involved transitions to various three-fold coordinated phases below $100 \mathrm{GPa}$. It has been suggested that the observed transition pressure can be substantially higher because of a large potential barrier to dissociation (e.g., Refs. [4,5]).

For transformation to framework structures, information about the type and character of the ordering is important, since it affects the energy barriers associated with the transition. Solid nitrogen is an important system for such studies because of the stability and simple electronic structure of the isolated molecule. Moreover, solid nitrogen has 
2

been well extensively studied theoretically, and accurate experimental data provide an important test of condensed matter theory [4-8].

The phase diagram of nitrogen (Fig. 1) is complex at moderate pressures and temperatures $[9,10]$ and until recently has been little studied over a wider range. The melting curve of $\mathrm{N}_{2}$ has been only measured to about $22 \mathrm{GPa}$ [11-13]. Higher P-T data are available from shock-wave CARS study [14], which refer to a liquid state. Solid nitrogen has a wealth of molecular phases that differ in the types of orientational ordering and crystal structures formed $[9,10,12,13,15-33]$. The ordering of the low pressuretemperature $\alpha$ and $\gamma$ phases is controlled by quadrupole-quadrupole interactions, whereas at higher pressures a class of molecular structures $\left(\delta, \delta_{\text {loc }}, \varepsilon, \zeta\right)$ stabilized by additional anisotropic intermolecular interactions is found [10]. The $\delta$ phase (Fig. 2) is proposed to have a disordered cubic structure (space group Pm3n) [22] with two different types of molecules exhibiting sphere - and disk- like disorder and giving rise to two classes of

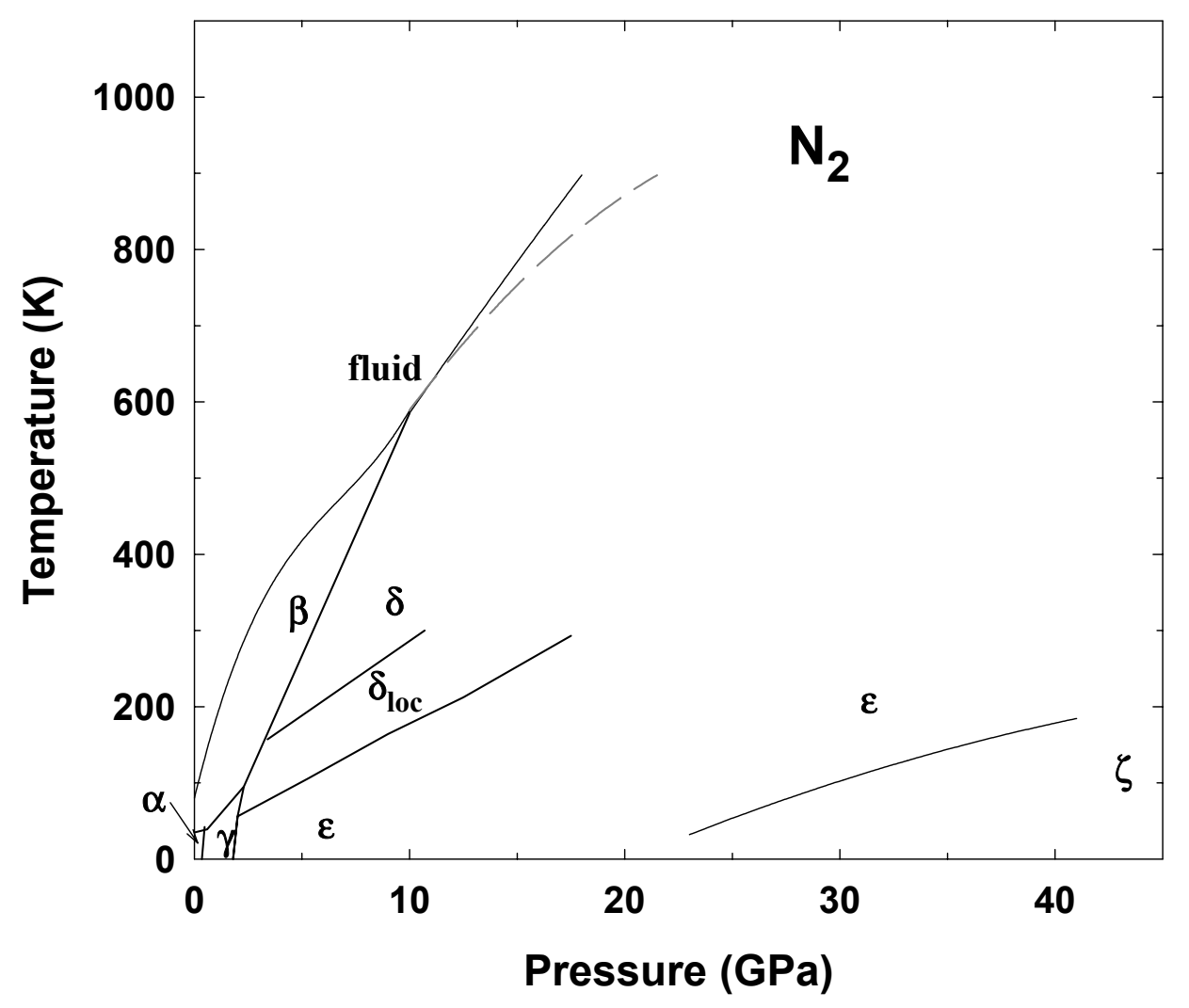

Fig. 1. A portion of the phase diagram of nitrogen at moderate pressure and temperature conditions $[9,11,12,25]$. Dashed gray line and thin solid line at high temperature correspond to the melting curve from Refs. [12] and [11], respectively .

vibron bands $v_{1}$ and $v_{2}$. With increasing pressure and/or decreasing temperature, nitrogen molecules exhibit orientational order through a sequence of the phase transformations (to $\delta_{\text {loc }}$ and then $\varepsilon$ ) as determined by vibrational spectroscopy and x-ray diffraction [27-36]. 
The structure of the $\varepsilon$ phase is rhombohedral (space group of $R \overline{3} c$ ), which can be viewed as a distortion of cubic [25].

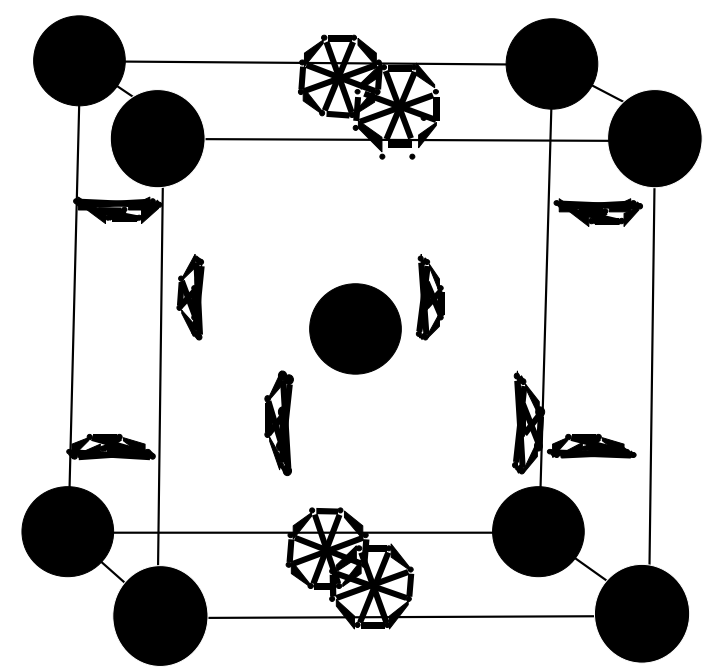

Fig. 2. The unit cell of $\delta-\mathrm{N}_{2}$ [22]. Black spheres are $\mathrm{N}_{2}$ molecules in sphere-like positions $(2 a$ site symmetry), black "wheels" are $\mathrm{N}_{2}$ molecules in disk-like positions ( $48 l$ site symmetry).

Theoretical calculations have been contradictory in their predictions of stable molecular ordered phases [6-8]. In particular, Monte Carlo (MC) [6] and Molecular Dynamics (MD) [7] simulations based on proposed intermolecular potential favor tetragonal structures, while ab initio calculations [8] predicted the stability of $R \overline{3} c$ phase in agreement with experiment. Recent MC calculations [31,32] pointed out the importance of using accurate atom-atom potentials (especially anisotropic terms) to obtain adequate results.

At 20-25 GPa and $10 \mathrm{~K}$ a transformation has been found by Raman spectroscopy [23] and confirmed by later Raman and IR measurements [9]. No x-ray data were available until recently for this phase (called $\zeta$ ). According to vibrational spectroscopy data, its structure has a strong similarity to $\varepsilon$ and $\delta$ phases. It has been inferred to have $R 3 c$ space group. The $\varepsilon-\zeta$ phase boundary has been extended recently to $180 \mathrm{~K}$ and $40 \mathrm{GPa}$ [9]. At low temperatures, a significant region of metastability is reported [9], making difficult to clarify the mechanism of the transformation.

At room temperature, the vibrational spectroscopy data are still contradictory about a number and nature of the transformations above $20 \mathrm{GPa}$. A sequence of new phases has been reported on the basis of several splitting of the Raman vibron modes [24], including one just above $20 \mathrm{GPa}$ [27]. In contrast, x-ray studies indicate the stability of $\varepsilon$ phase to $50 \mathrm{GPa}[26,33]$ in agreement with latter Raman study [34]. A change in x-ray diffraction pattern was observed above $60 \mathrm{GPa}$ [36], but interpretation requires additional measurements. Recent Raman and IR measurements to $42 \mathrm{GPa}$ show clear correspondence between the number of observed lattice and vibron modes and grouptheoretical predictions for the $\varepsilon$ phase [9]. 
Here we review our recent combined optical, Raman, synchrotron IR and x-ray diffraction high-pressure studies of nitrogen up to $270 \mathrm{GPa}$ between 10 and $300 \mathrm{~K}$ and temperatures above $1000 \mathrm{~K}$ at pressures up to $150 \mathrm{GPa}$. [37-40].

\section{EXPERIMENTAL}

Pressure was generated with a diamond anvil cell (DAC) employing beveled anvils with central flats ranging from 20 to $100 \mu \mathrm{m}$ and flat diamonds with 200-500 $\mu \mathrm{m}$ culets. Two types of DAC were used: modified (to match a continuous flow He cryostat) Mao-Bell cell for operations at room and low temperatures [41] and a Mao-Bell high-T external heating cell [42]. The latter one is equipped with two heaters and thermocouples. Four experiments were performed at RT aiming to highest pressure and the final pressures varied from 180 to $268 \mathrm{GPa}$. For low-temperature measurements we used a continuousflow He cryostat, which allowed infrared and in situ Raman/ fluorescence measurements. More details about our IR/Raman/fluorescence setup at the NSLS are published elsewhere [41].

To explore high P-T transformations of nitrogen, we performed more than 20 experiments ranging from 15 to $1050 \mathrm{~K}$ and up to $150 \mathrm{GPa}$ using in situ Raman spectroscopy and also synchrotron infrared (IR) spectroscopy and x-ray synchrotron diffraction on temperature-quenched samples. For Raman spectroscopy we used 514.5 and $487.9 \mathrm{~nm}$ and tunable red lines of a Ti:Sapphire laser as excitation sources. The temperature was measured to within $\pm 1 \mathrm{~K}$ below $600 \mathrm{~K}$ and $\pm 5 \mathrm{~K}$ above $600 \mathrm{~K}$. To determine the pressure, we used in situ fluorescence measurements of ruby and Sm:yttrium aluminum garnet chips loaded in the sample chamber. Energy dispersive xray diffraction was carried out at the beam line X17C of the National Synchrotron Light Source (NSLS), using a focused white beam. To reduce possible texture problems the sample was rocked in $\mathrm{v}$ and $\mathrm{x}$ over an angular range allowed by the diamond backing plates. The majority of the diffraction data, however, were collected at the 13-ID beam line of GeoSoilEnviroCARS (GSECARS) at the Advanced Photon Source (APS). A focused, monochromatic beam at $0.4246 \AA(29.2 \mathrm{keV})$ was used, and the data were recorded on a MAR charge coupled device calibrated with a $\mathrm{CeO}_{2}$ standard. The sample was rocked in $\mathrm{v}$ by $\pm 10^{\circ}$ to average over as many crystallites as possible.

\section{ORIENTATION ORDER IN MOLECULAR PHASES}

Fig. 3 shows Raman spectra as a function of pressure. As was discussed above, the splitting of the vibron in $\delta-N_{2}$ (not shown, see e.g. Refs. [20]) and $\varepsilon-N_{2}$ to $v_{1}$ and $v_{2}$ components manifests the presence of sites of different site symmetry (disk $48 l$ and sphere-like $2 a$ ). With pressure, the $v_{2}$ mode further splits successively to several components. The first splitting corresponds to the delta-epsilon transition at $18 \mathrm{GPa}$. The second component is first seen as a weak shoulder on the high-frequency side of the major component. The splitting occurs due to the reduction of symmetry (monoclinic distortion), so differently polarized vibrations have different symmetry and may have different energies. An additional splitting of the main component is related to phase transformations due to a sequential orientational ordering of molecules, as will be discussed later. Additional and very important information about orientational ordering can be obtained from the spectra of the translational (phonons) and rotational (librons) vibrations (see below). 
Qualitatively similar phenomena are observed are low temperatures, but the transformations are more pronounced and occur at lower pressures. The sequence of Raman spectra measured as a function of pressure at low temperatures is shown in Fig. 4. At $17.5 \mathrm{GPa}$ we observe two Raman peaks in the vibron region -slightly broadened $v_{2}$ (lower frequency) and $v_{1}$. This is in excellent agreement with the experiments for the $\varepsilon$ phase (see also Refs. $[9,23]$ ). Increasing pressure splits the $v_{2}$ peak, so three components can be seen. This splitting becomes obvious at the highest pressure (44 GPa), while at 24$38 \mathrm{GPa}$ peak fitting is required to reveal the two components (e.g. the spectrum at 24.8 $\mathrm{GPa}$ in Fig. 4). Only a slight broadening of the $v_{1}$ peak is observed as the pressure is increased. Lattice modes also change at 18-25 GPa, revealing splitting of the major bands

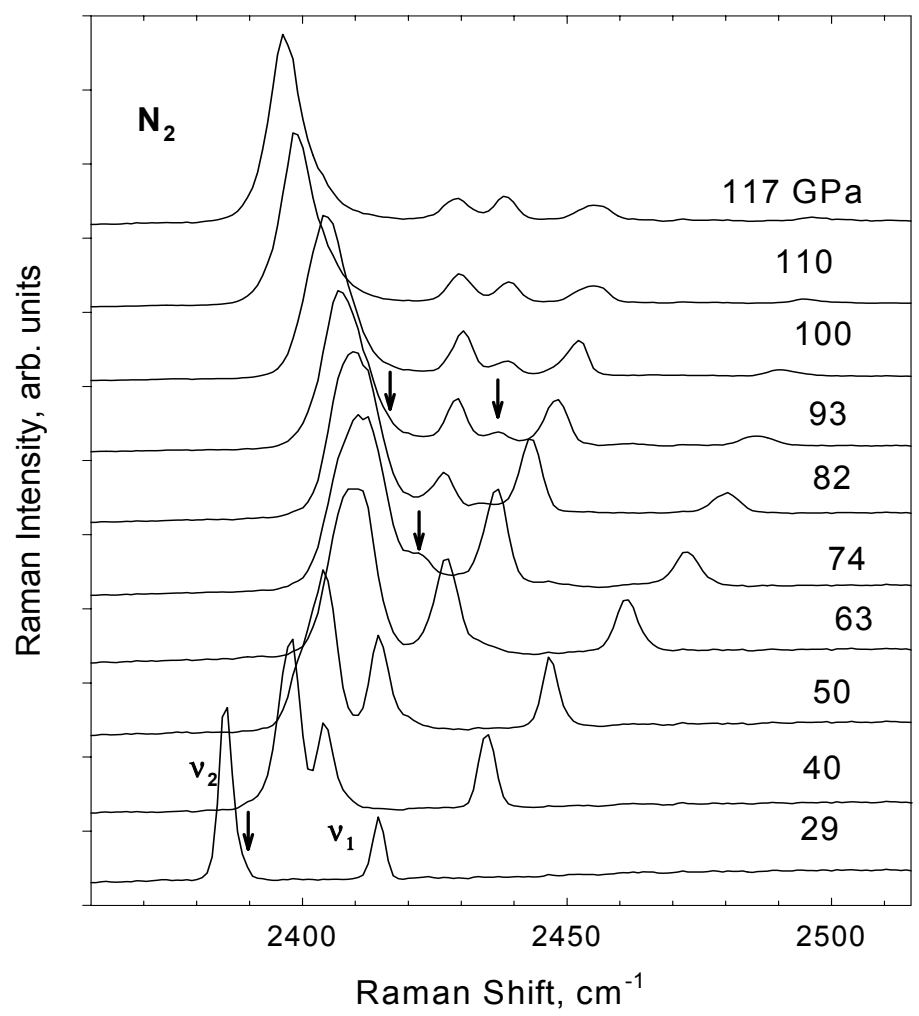

Fig. 3. A sequence of Raman spectra of $\mathrm{N}_{2}$ as pressure increases at room temperature. Vertical arrows indicate new bands, which appear as pressure increases.

and appearance of new bands (Fig. 5) in agreement with previous studies for $\varepsilon-\mathrm{N}_{2}[9,23]$.

Infrared spectra at $17.5 \mathrm{GPa}$ (Fig. $4 \mathrm{~b}$ ) show a very weak absorption in a spectral range of the $v_{2}$ vibron. The absorbance increases substantially at $18-25 \mathrm{GPa}$, so a doublet of IR vibrons is clearly visible at higher pressure. As for the Raman bands, a moderate broadening of the IR peaks is observed as the pressure is increased.

The pressure dependence of the observed Raman frequencies is shown in Fig. 6. Corresponding data from Ref. [9] are also shown for comparison. Earlier results [23] for the $v_{2}$ multiplet are very close to those reported in Ref. [9] and are not shown for clarity. For the $v_{1}$ band, our low-temperature data are very close to those measured at the room temperature (see also Ref. [32]). 
6

The substantial difference in the pressure dependence of the frequency of the Raman $v_{2}$ multiplet will be discussed later. The pressure dependences of the Raman and infrared frequencies are compared in the inset of Fig. 6. We find that Raman and infrared frequencies do not coincide (cf. Ref. [9]) suggesting a non-centrosymmetric space group. Unlike the Raman data, our infrared frequencies are in good agreement with those reported in Ref. [9] in the pressure range overlapped by the two studies (to 25.2 GPa).

At room temperature, Raman spectra of $\mathrm{N}_{2}$ measured above $60 \mathrm{GPa}$ (Fig. 3) contain more vibron bands (four) that are allowed by group theory for the R 3 c symmetry of $\varepsilon-\mathrm{N}_{2}$ (three) [23]. Increasing of pressure through the $60 \mathrm{GPa}$ range gives rise to a new Raman peak $\left(v_{2 b}\right)$, designated by arrow in Fig. 3 (see also Refs. [24,35]). A similar observation was made when cooling down from approximately the same starting point [36]. At this pressure (and room temperature) we also observed an increase in intensity of the infrared vibron [3]. The splitting of the lattice modes increases with pressure with multiplets evolving into distinct bands.
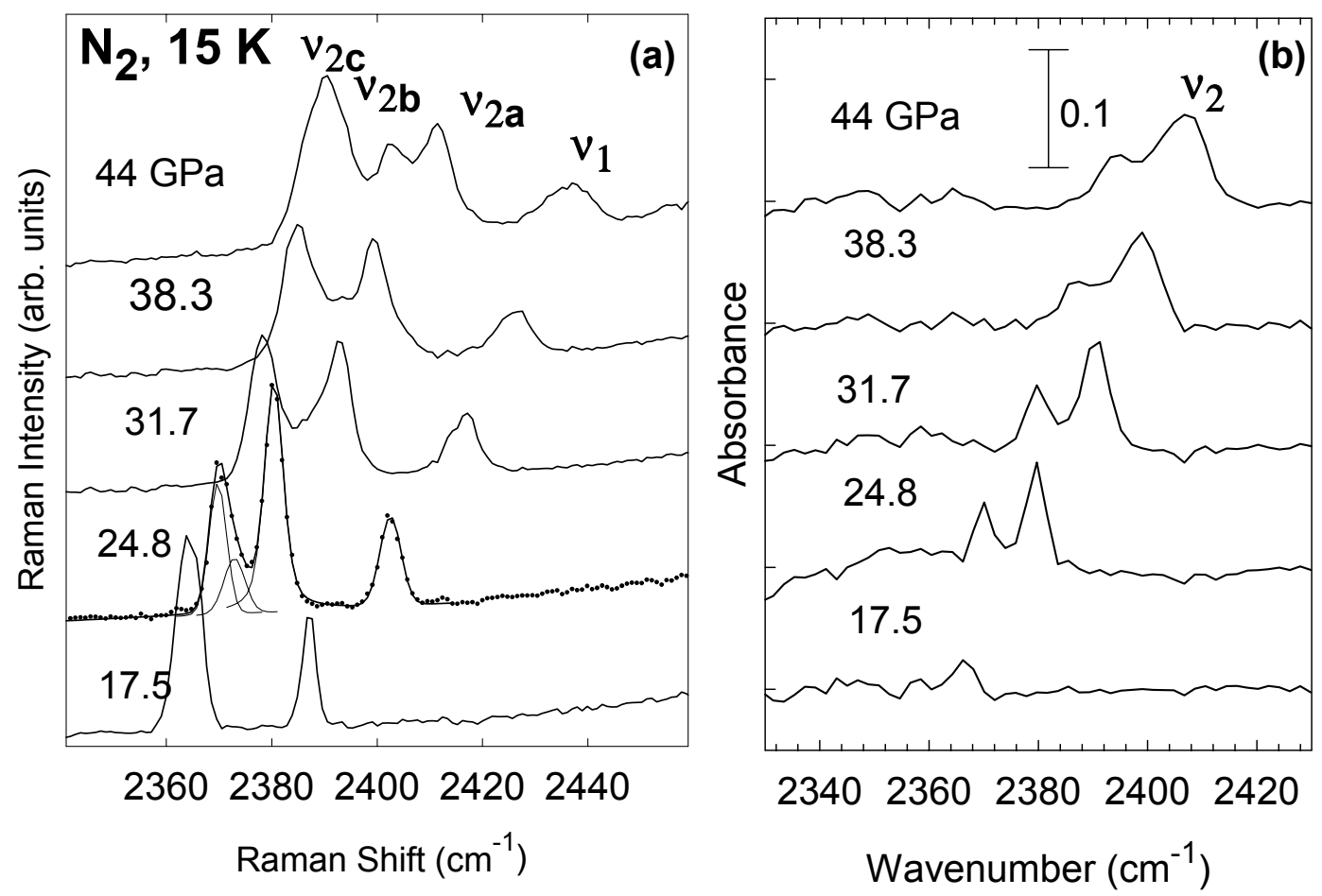

Fig. 4. Raman (a) and IR (b) spectra of nitrogen through the low-temperature transition to the $\zeta$ phase. The spectra are shifted in vertically for clarity. The Raman spectrum at $24.8 \mathrm{GPa}$ (points) is shown along with the fitting to multiple components (Voigt profiles).

Raman spectra to $140 \mathrm{GPa}$ (Fig. 3) reveal an abundance of vibrational modes in agreement with previously reported results $[24,27,35]$. At moderate pressures, our data are in agreement with those reported in Refs. [24,27,35]. As in Refs., [24,27,35], we observe branching of the Raman-active vibrons at around $90 \mathrm{GPa}$ followed by an increase in their frequency separation. Intensity redistribution is also observed as the lowest frequency vibron $v_{2}$ gathers most of the intensity at the highest pressures. As in Refs. $[24,35,45]$, we also observe a softening of the low-energy Raman vibron, which was 
initially interpreted as an indication of approaching dissociation of $\mathrm{N}_{2}$ molecules. In contrast, we find a monotonic increase in frequency (Fig. 5) of all lattice modes and redistribution of their intensities with pressure, so that high-frequency bands dominate at the highest pressures.

IR absorption spectra (see Fig. 7) reveal a multiplet of modes in the vibron spectral range and also a newly discovered mode of lattice vibrations (see below). The pressure dependencies of IR vibron frequencies match well the extrapolation of the lower pressure data reported in Refs. [44,45]. The pressure dependence of the lattice mode frequency is very close to that of the high-frequency Raman lattice mode (Fig. 8). As in the case of Raman vibrons, the IR vibrons show branching with pressure, so that up to 5 vibrons can be observed at high pressure, all of which originate from the $v_{2}$ multiplet. The Raman and IR vibrons probe different points of the large Brillouin zone and become accessible because of its folding. The lowest-frequency Raman vibron corresponds to the lowest frequency of the Brillouin zone and represents the case where all molecules on the faces of the unit cell (e.g. Ref. 27) vibrate in phase [37]. The other vibrons (Raman and IR) involve different out-of-phase vibrations and form a compact group close to the frequency of the uncoupled N-N stretch. The splitting between the highest and lowest frequency vibrons approximately quantifies the Brillouin zone bandwidth, which increases dramatically with pressure [37]. The frequency of the uncoupled N-N stretch tentatively determined near the position of the density maximum of the observed vibrons

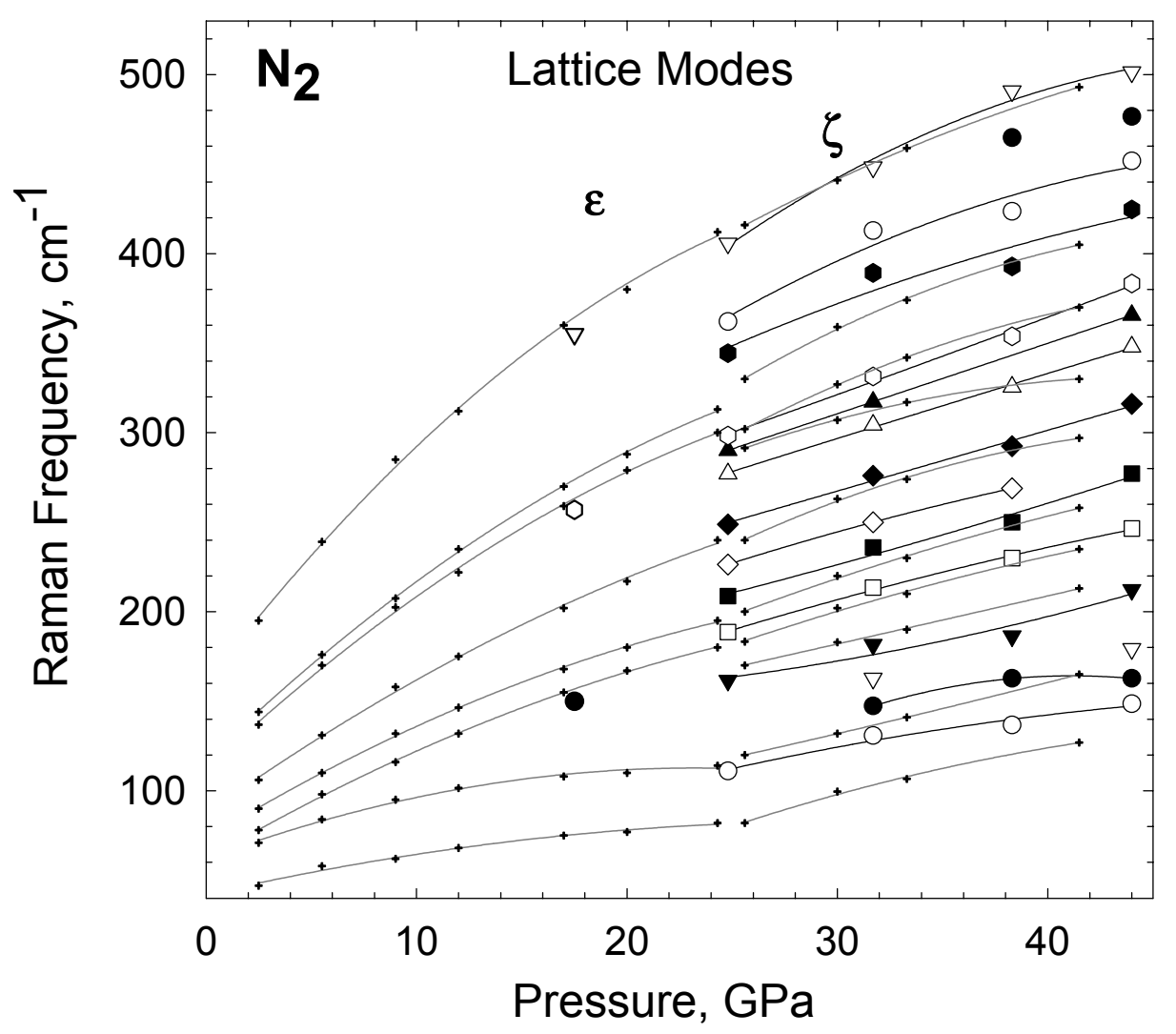

Fig. 5. Raman frequencies of the lattice modes at low temperatures as a function of pressure. Large symbols and solid lines are our data. Dots and grey lines (square fittings) are data from the Ref. [9]. 
(Fig. 7) levels off in the 100-150 GPa pressure range. Thus, the turnover and softening with pressure of the lower-frequency Raman vibron is naturally explained by an increase in the vibrational splitting, which in turn is caused by an increase in the intermolecular interactions. The observed redistribution of intensities between lattice and vibron modes can be considered a consequence of mixing of vibrational states arising from the difference in crystallographic positions at the unit cell face and origin. This means that nitrogen molecules are sitting in quasi-homogeneous sites prior to the transition to a nonmolecular state unlike another possible scenario involving formation of heterostructures [46].

At pressures above $90 \mathrm{GPa}$ and room temperature a new Raman vibron band $\left(\mathrm{v}_{2 \mathrm{a}}\right)$ become visible (see also, Ref. [35]). At this point the infrared absorption spectra of vibron modes also show a change: more IR modes become visible and the overall intensity increases (Fig. 7). Phase transition has been suggested in Ref. [24] on the basis of similar observations, but it was not confirmed in more recent publication [35]. The data presented here and also the results of the recent unpublished x-ray study (Sanloup et al., unpublished) clearly indicate a phase transformation at 100-110 GPa. This transition may be related to a next (probably ultimate) stage of orientation ordering of $\mathrm{N}_{2}$ molecules.

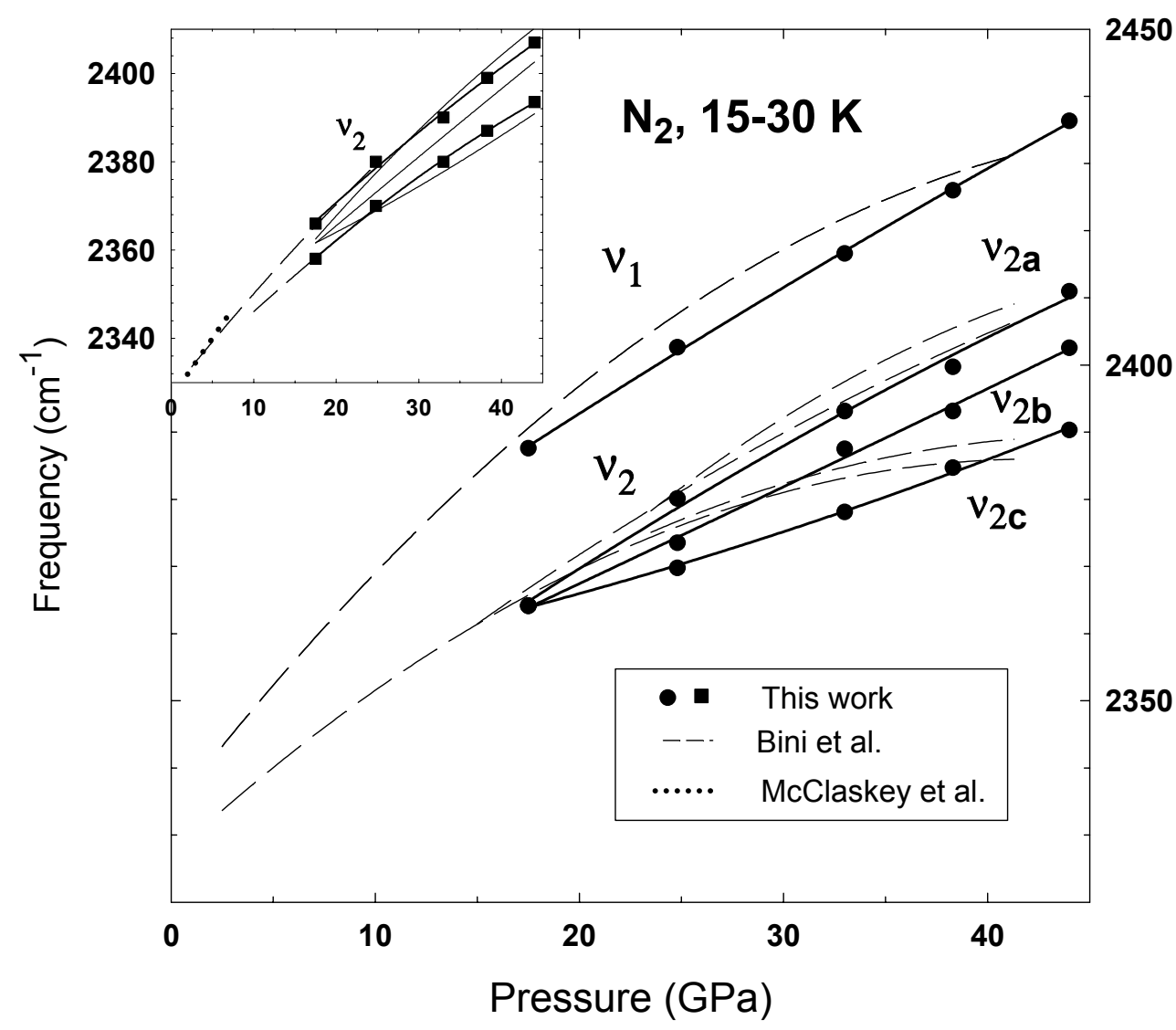

Fig. 6. Raman vibrational frequencies as a function of pressure at $15 \mathrm{~K}$. Filled circles are the Raman data from this work. The solid thick solid lines are the guides to the eye. The dashed lines are the Raman data from Ref. [9]. The inset shows the comparison between Raman and infrared frequencies. Filled squares are the infrared data from this work. The solid thick solid lines are the guides to the eye. Thin solid lines are the Raman data from this work. 


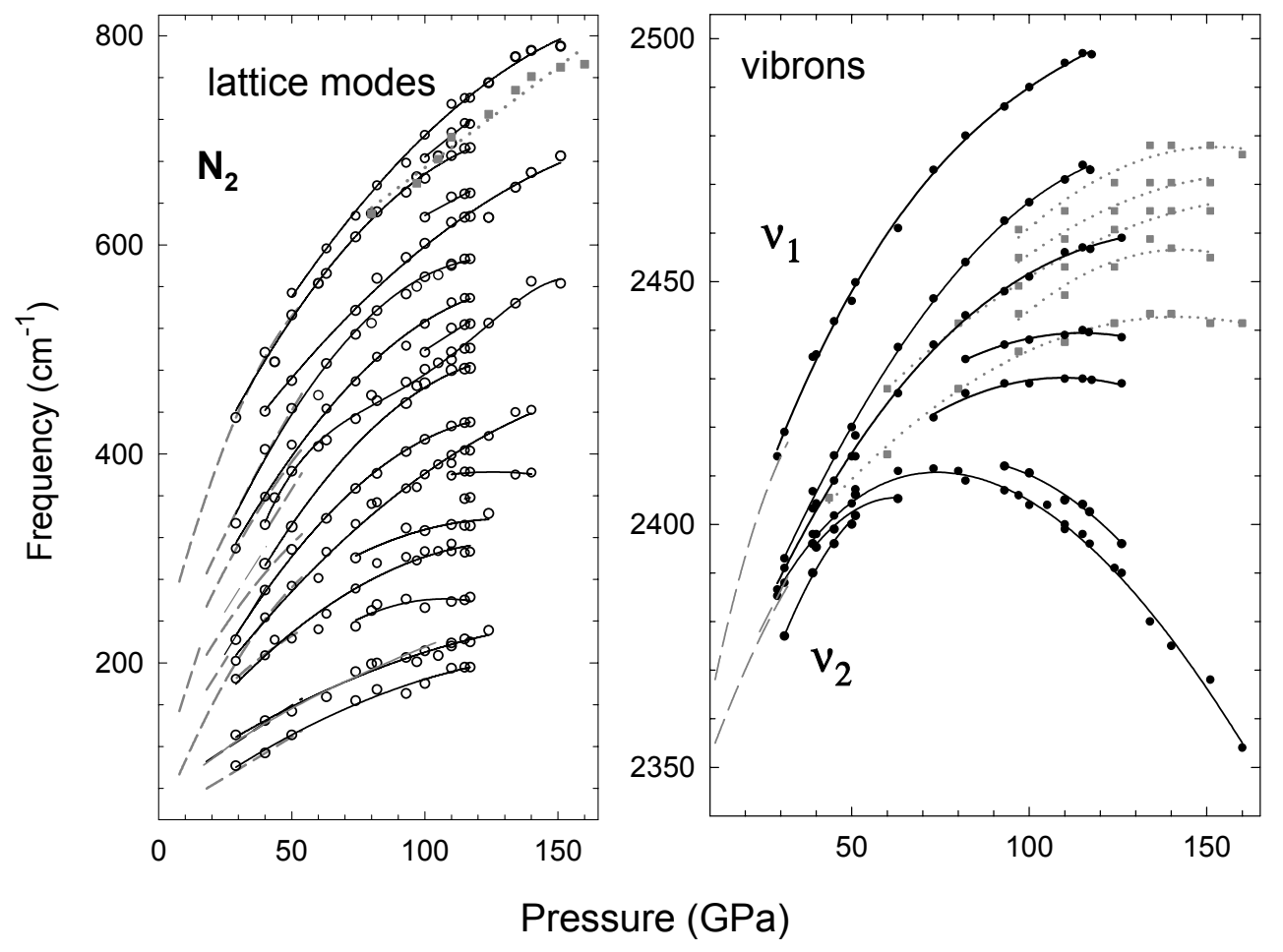

Fig. 7. Raman and infrared lattice and vibrational frequencies of $\mathrm{N}_{2}$ at high pressures and room temperature. Circles - Raman data, squares - IR data. Solid line and dotted grey lines are guides to the eye for these data, respectively. Grey dashed lines are from Ref [27].

The splitting of the vibron bands and change in the lattice mode spectrum observed at low temperature indicate a phase transformation related to orientational ordering of the nitrogen molecules. The phase diagram of Ref. [9] suggests that these changes correspond to the $\varepsilon \rightarrow \zeta$ transition. Qualitatively, our data and those presented in Refs. $[9,23]$ show similar trends, but detailed comparison shows different Raman spectra for the high-pressure phase (Fig. 6). We believe that the disagreement arises from the use of different experimental procedures and the nature of the high-pressure phase (or phases). In contrast to experiments reported in Refs. [9,23], we changed pressure at low temperature. It is useful to note that when infrared spectra were measured in a manner similar to ours [9], the results from both studies agree very well (inset to Fig. 3). The evidence that the properties of the high-pressure phase depend on the thermodynamic path, suggests that this phase is not thermodynamically stable (i.e. metastable at the indicated P-T conditions). This is supported by observations of a large hysteresis of the transition at low temperatures [9]. An alternative (but related) explanation is that different properties of the high-pressure phase arise from relatively large pressure inhomogeneities in our experiment (since we changed pressure at low temperature) as indicated by broadening of Raman and infrared bands at higher pressures (Fig. 4).

The changes in Raman and infrared spectra above $60 \mathrm{GPa}$ at room temperature are very similar to those observed at low temperatures. Moreover, the reported transition boundary 
10

[9] extrapolated to high pressure and temperature matches this room-temperature point. According to the observed Raman and infrared spectra, the vibrational properties of the high-pressure phase are very similar at room and low temperature. Thus, we will consider it to be the same phase $(\zeta)$.

In view of the absence of sufficient $x$-ray data for the $\zeta$ phase, we can only speculate on its crystal structure. The number of the vibron modes (in either our experiment or those reported in Ref. 35) exceeds that predicted for the $R 3 c$ structure based on the space group theory proposed in Ref. [23]. According to Ref. [35], the increase of number of vibron modes is due to the increase in the number of different site symmetries occupied by $\mathrm{N}_{2}$ molecules. Following this idea, up to 5 different site symmetry positions should be invoked to explain the observed number of Raman vibron peaks above $60 \mathrm{GPa}$, which does not seems plausible. A critical examination of the spectra of Ref. [35] shows that this number can probably be reduced to 3 according to the number of observed distinct peaks in the Raman excitations of the guest molecules. Thus, it seems natural to propose that the branching of vibron modes is related to sequential lifting of degeneracy of the $v_{2}$ term of the cubic $\delta$ phase. In the first stage ( $\delta$ to $\varepsilon$ transition), the $v_{2}$ band splits into $A_{1 g}$ and $\mathrm{E}_{\mathrm{g}}$ components by the crystal field. In the second one ( $\varepsilon$ to $\zeta$ transition), the symmetry is further reduced (to orthorhombic or monoclinic), with doubly degenerate level splitting into two components. Additional splitting (vibrational or Davydov-type) of these major components could be caused by intermolecular interactions. This is related to a possible increase in the number of molecules in the unit cell as well as associated symmetry lowering. High-quality diffraction data are required to examine these hypotheses.

We find that the properties of the high-pressure, low-temperature phase of nitrogen obtained by "cold" compression are different from those for the phase quenched from high temperature. This suggests that the $\zeta$ phase is metastable and/or transitions to it are sensitive to nonhydrostatic effects. Raman and infrared spectra of $\varepsilon-\mathrm{N}_{2}$ above $40 \mathrm{GPa}$ and $\zeta-\mathrm{N}_{2}$ are not compatible with $R \overline{3} c$ and $R 3 c$ symmetries proposed in Ref. [23] because the number of vibron bands is larger than predicted for the standard structures based on these space groups. This increase in the number of bands is probably related to the additional lowering of the symmetry and multiplication of the size of the unit cell.

The present vibrational spectroscopy data provide additional constraints on the structure and properties of the high-pressure phases. They also suggest that known phases are not necessarily thermodynamically stable in the P-T region in which they can be observed. As for other molecular crystals, sluggish kinetics can complicate the determination of the true thermodynamic phase diagram (see e.g. Ref. [47]). Further theoretical and experimental effort is necessary to obtain a better understanding of the phase diagram of nitrogen at these high-pressure conditions.

\section{NEW CLASSES OF MOLECULAR PHASES}

When compressed at $300 \mathrm{~K}$ nitrogen transforms from the $\delta$ to $\zeta$ phase around $60 \mathrm{GPa}$ (see Fig. 11). When heated at pressures higher than $60 \mathrm{GPa}$ the material first back transforms from $\zeta$ to $\varepsilon$ along a boundary that we find to be on the extension of the line established in Ref. 9 at lower temperatures. At $95 \mathrm{GPa}$ when the temperature reaches $>600 \mathrm{~K}$, the transition to $\theta$ nitrogen takes place. The transition can be observed visually since $\varepsilon(\zeta)-\mathrm{N}_{2}$ normally shows substantial grain boundaries, while after the transition to the $\theta$ phase, the sample looks uniform and translucent. In most cases the transition happens instantaneously and goes to completion within seconds as determined by Raman 
spectroscopy. If $\varepsilon-\mathrm{N}_{2}$ is heated at even lower pressures (e.g., 65-70 GPa), it transforms above $750 \mathrm{~K}$ to $l-\mathrm{N}_{2}$. It is also possible to access the 1 phase from $\theta$ : we observed the transformation from the $\theta$ to $\mathrm{l}$ phase on pressure release at $850 \mathrm{~K}$ at $69 \mathrm{GPa}$ (see below).

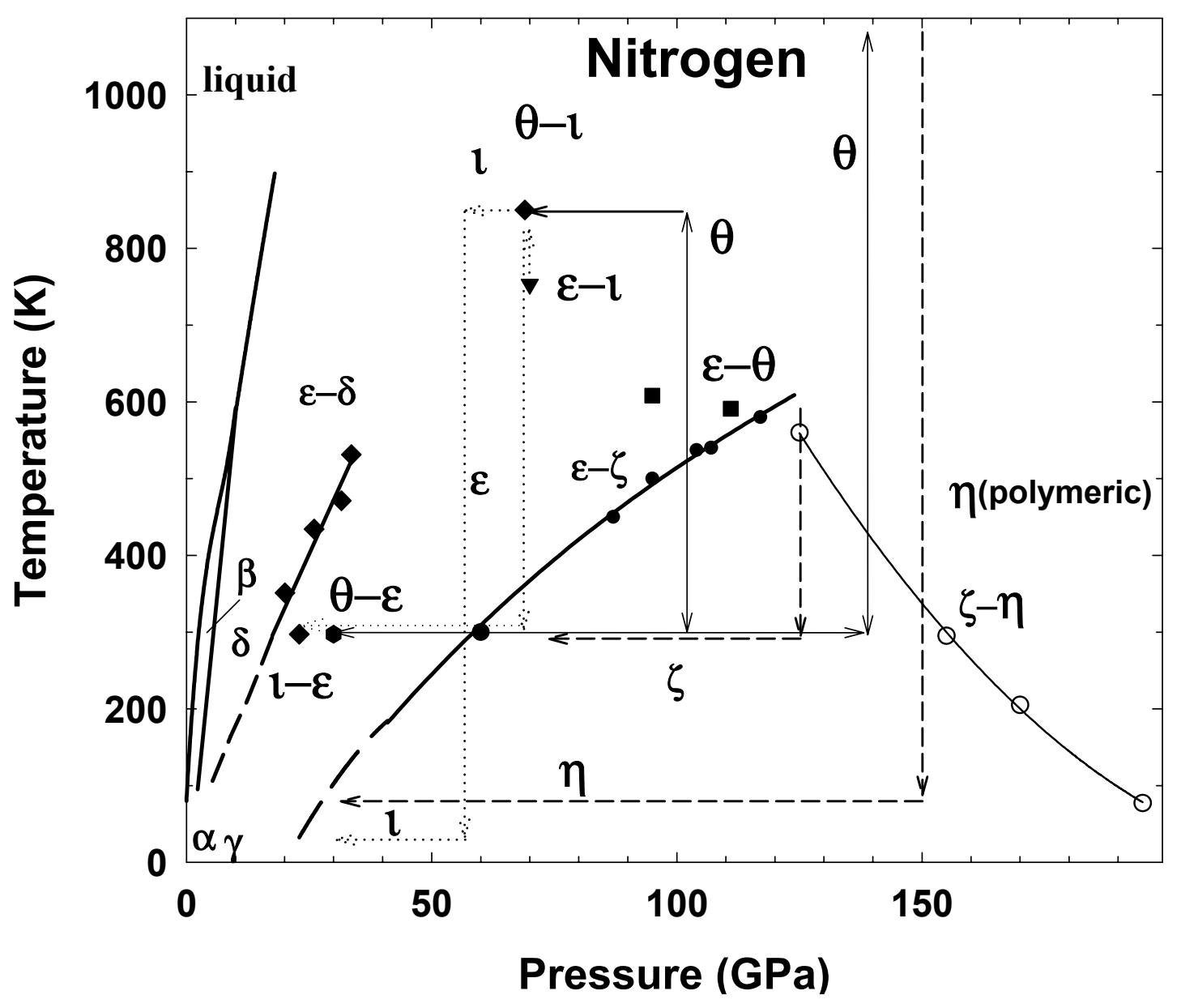

Fig. 8. Phase and reaction diagram of nitrogen at high pressures and temperatures. Solid thick lines are thermodynamic boundaries. Solid circles show the transitions between $\delta-\varepsilon$ and $\varepsilon-\zeta$ phases investigated in this work. Filled symbols ( 1 - diamonds, $\theta$ - squares) show the $P$ - $T$ points at which new phases were reached or back transformed to the known phases. The arrows show thermodynamic paths (schematic) used to reach $\theta$ (solid, thin lines) and 1 (dashed, thin lines) phases and paths taken to investigate their stability. The transformation to nonmolecular $\eta$-nitrogen is shown by the open circles (this work and Ref. 48) and thick solid line, which is only a guide to the eye. This region should be treated as a kinetic boundary. Phase boundaries at low $P-T$ (open squares) are from Ref. 9 and the melting curve is from Ref. 11. The phase boundaries for the $\alpha, \gamma$, and $\delta_{l o c}$ phases are not shown.

Figure 9 shows the Raman spectra of the $\imath$ and $\theta$ quenched to room temperature. In order to have spectra of all phases discussed here at similar conditions we present them at room temperature. The high-temperature spectra of $\imath$ and $\theta$ are very similar to ones measured upon quenching the sample to $300 \mathrm{~K}$. All IR measurements were performed at $300 \mathrm{~K}$. The Raman and IR spectra of the $\zeta$ phase (obtained by compression at $300 \mathrm{~K}$ ) from which 
12

these phases were formed are shown for comparison at $69 \mathrm{GPa}$ (Fig. 9) and $97 \mathrm{GPa}$ (Fig. $10)$.

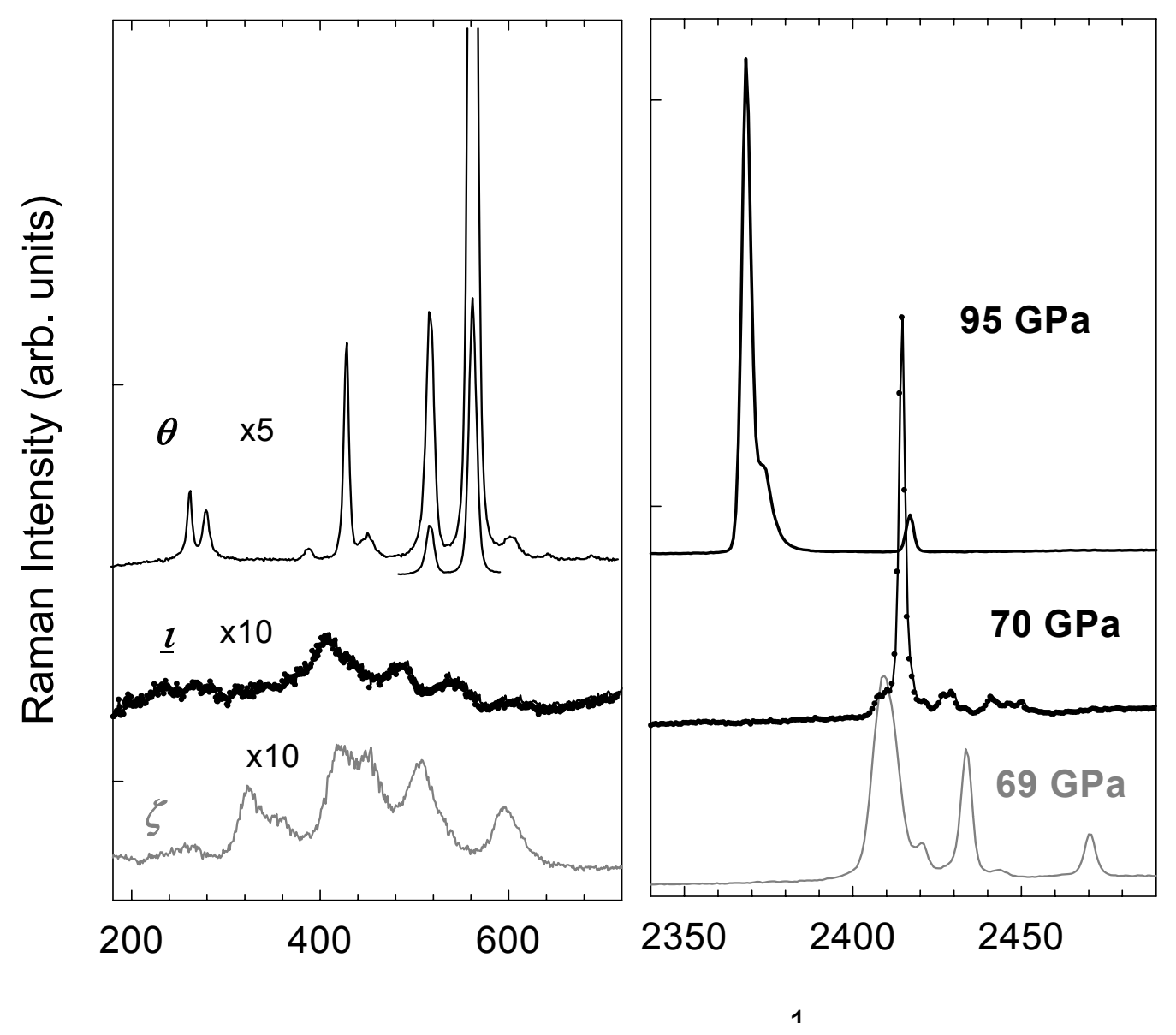

Fig. 9. Representative Raman spectra of $\theta$ and $\imath$ phases measured at 95 and $70 \mathrm{GPa}$ and $297 \mathrm{~K}$ after quenching from high temperature. The spectra of the $\zeta$ phase used as a starting material are shown for comparison at the same temperature and at $69 \mathrm{GPa}$.

Raman spectra of both $\theta$ and $\mathrm{l}$ exhibit vibron excitations (Fig. 9, right panel), although their number and frequencies differ from those of all other known molecular phases. The changes in the low-energy region of the spectra (Fig. 9, left panel) for $\theta$ phase are very pronounced. The lattice modes of $\theta$ nitrogen are very sharp and high in intensity compared to either 1 or $\zeta$ (also $\varepsilon$ ). This is a clear indication that molecular ordering in $\theta$ is essentially complete, whereas other molecular phases may still possess some degree of static or dynamic orientational disorder. The lattice modes of $1-\mathrm{N}_{2}$ are also different from those in $\zeta$ and $\varepsilon$ : they are extremely weak and broad, suggesting that this phase is not completely orientationally ordered.

Figure 10 shows infrared-absorption spectra of new phases together with that of the $\zeta$ phase. Again, number, frequencies, and intensities of vibron excitations (Fig. 10, right 
panel) are different from other known phases. In the case of $\mathrm{t}-\mathrm{N}_{2}$ the spectrum differs mainly in positions of the absorption bands while the IR vibron mode of the $\theta$ phase has a much larger oscillator strength compared to other $\mathrm{N}_{2}$ phases $\mathrm{cf.} \mathrm{H}_{2}$ in phase III (Ref. [49]) and $\varepsilon-\mathrm{O}_{2}$ (Ref. [50]). There is a more pronounced Raman and IR softening of the vibron bands of $\theta$ nitrogen compared to the other modifications $(1, \zeta$, and $\varepsilon$ ). This observation together with the presence of a strong IR vibron is consistent with the existence of charge transfer e.g., see Refs. [49-51] related to the formation of lattice-induced dipole moments or association of molecules [50-52].

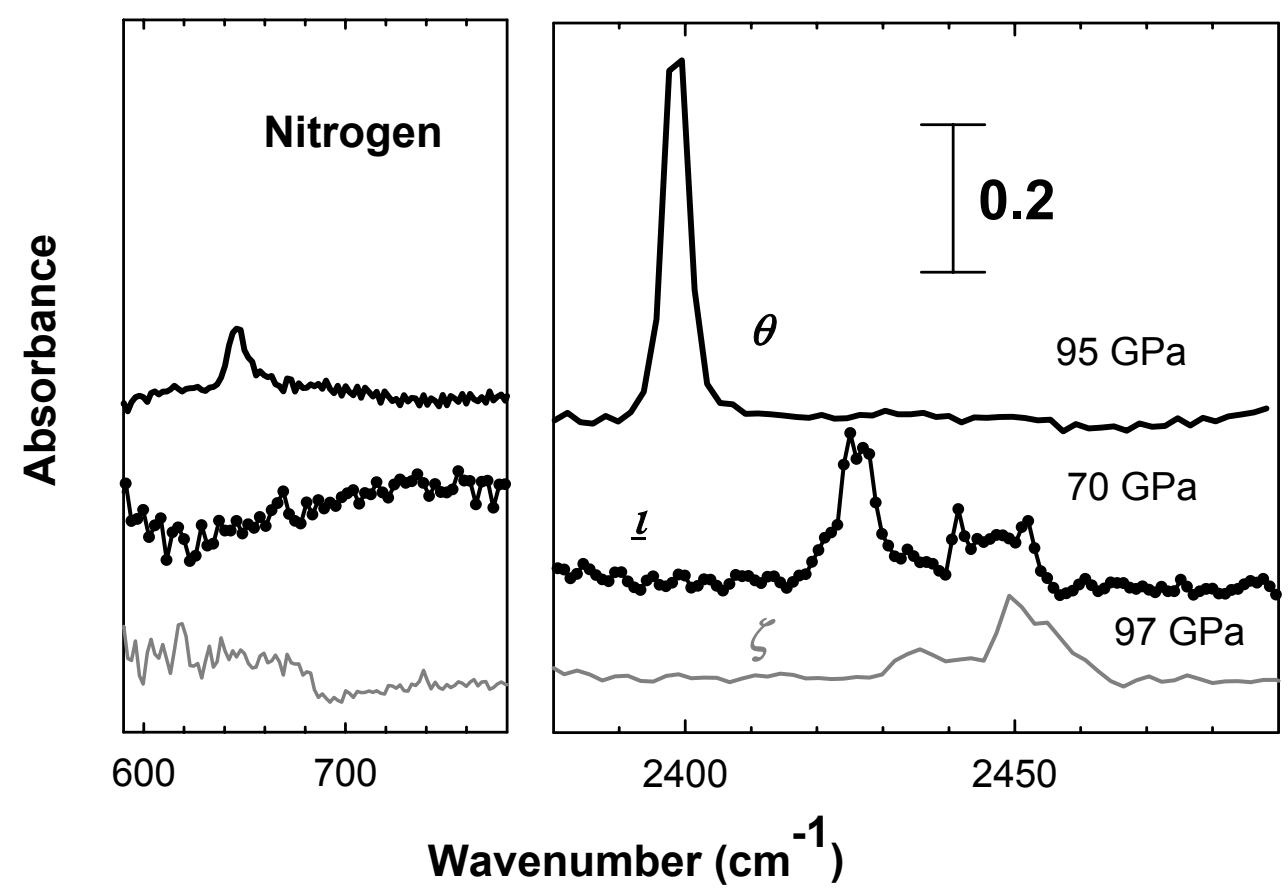

Fig. 10. Infrared modes of $\theta$ and $\mathrm{l}$ phases measured at 95 and $70 \mathrm{GPa}$ and $297 \mathrm{~K}$ after quenching from high temperature. The spectra of the $\zeta$ phase used as a starting material were measured at $97 \mathrm{GPa}$ and are shown for comparison at the same temperature.

The pressure dependence of the Raman-active vibron modes (Figs. 11(a) and 11(b)) was studied on unloading at $300 \mathrm{~K}$ in new phases. $\mathrm{l}-\mathrm{N}_{2}$ exhibits typical behavior for such molecular crystals: branching of vibrational modes and increasing of separation between them with pressure due to increasing intermolecular interactions. All of the vibrational modes originate from the same center, which is close to the frequency of the $v_{2}$ disk-like molecules in $\varepsilon-\mathrm{N}_{2}$. Thus, the structure of the 1 phase is characterized by the presence of just one type of site symmetry for the molecules and the large number of vibrational modes arises from a unit cell having a minimum of eight molecules. For the $\theta$ phase, two different site symmetries are present. The higher frequency $v_{1 \theta}$ gives rise to three Raman bands and one in the IR, while the lower frequency $v_{2 \theta}$ correlates with only one Raman band. Figures 11(a) and 11(b) also show that the spectra have several cases of frequency coincidence of Raman and IR vibron modes, which excludes an inversion center for both structures. Surprisingly, we find that the $\theta$ and 1 phases have a very large domain of pressure and temperature metastability. The pressure dependence of the Raman spectra (Fig. 11) was studied in a wide pressure range at $300 \mathrm{~K}$ in both phases. Most data were taken on pressure release, although experiments carried out on different conditions did not reveal that any measurable differences in frequencies of the $\theta$ and $\iota$ phases depended 
14

on the sample history. Performing x-ray analyses on low-Z materials in the $100 \mathrm{GPa}$ pressure range and high temperatures is difficult due to the low scattering efficiency

Fig. 11. (a) Raman (open circles) and infrared (open squares) frequencies of vibron modes as a function of pressure for 1 phase. (b) Raman (open circles) and infrared (open square) frequencies of $\theta$ phase. All measurements were done on the pressure release at $300 \mathrm{~K}$. Filled circles correspond to the vibron frequencies after the transformation to the $\varepsilon$ phase from $\theta$ and $\imath$ phases. Gray lines are data for the $\varepsilon$ and $\zeta$ phases from Ref. 35.

and small openings in the diamond backing plates, leading to low signal/noise ratios. Nevertheless, we performed synchrotron x-ray diffraction to confirm the existence of two new structures. First, we found good agreement with previously reported results for the low-pressure phases [26,33]. Only a few reflections could be observed above $50 \mathrm{GPa}$ because of strong sample texture. The highly textured nature of the sample could result in substantial changes in intensities of diffraction peaks from an ideal powder and even prevent observation of some of them. For the $\theta$-phase results presented here, we combined the energy- and angle-dispersive measurements for three samples with presumably different preferred orientations of crystallites. No major changes in the x-ray diffraction patterns were observed at $60 \mathrm{GPa}$ and room temperature, corresponding to the $\varepsilon-\zeta$ transition (see also Ref. 36). This observation is consistent with vibrational spectroscopy, which shows only moderate changes identified as a further distortion of the cubic unit cell of the $\delta$-phase [24,39]. In contrast, the x-ray-diffraction patterns of the samples after $\varepsilon \rightarrow \theta$ and $\varepsilon \rightarrow \imath$ transformations differ substantially from those of the $\varepsilon$ and $\zeta$ 
phases, and from each other. Tentative indexing of the peaks of $\theta$ nitrogen gives an orthorhombic unit cell e.g., with lattice parameters $a=6.797(4), b=7.756(5)$, and $\mathrm{c}=3.761(1) \AA$ at $95 \mathrm{GPa})$. The systematic absences, lack of inversion center and presence of high-symmetry sites (see above) are consistent with space groups Pma2, Pmn $2_{1}$, $P m c 2_{1}, P n c 2$, and $P 2_{1} 2_{1} 2$. The a/c ratio is close to $\sqrt{3}$, which suggests that the lattice is derived from a hexagonal structure. Extrapolation of the equation of state of $\varepsilon-\mathrm{N}_{2}$ measured to $40 \mathrm{GPa}$ (Ref. 26) shows that volume for this phase is about $14 \AA^{3} /$ molecule at $95 \mathrm{GPa}$, which gives an upper bound assuming a pressure-induced (density driven) transition. Comparison with the experimentally determined unit-cell volume $\left(198 \AA^{3}\right)$ suggests 16 molecules in the unit cell, giving $12.4 \AA^{3} /$ molecule in the $\theta$ phase and an $11 \%$ volume collapse at the $\varepsilon-\theta$ transition. The number of molecules is in agreement with vibrational spectroscopy data, although it is possible to describe the vibrational spectra with a smaller number (up to 8).

In order to better understand the P-T provenance of new phases and their relation with other polymorphs of nitrogen (Fig. 8), we pursued extensive observations in different parts of the phase diagram. The new phases are found to persist over a wide P-T range. As noted above, both phases could be quenched to room temperature. On subsequent heating, the $\theta$-phase remained stable when heated above $1000 \mathrm{~K}$ between 95 and 135 $\mathrm{GPa}$ and does not transform to the nonmolecular hphase (shown by arrow in Fig. 8). But on pressure release it transforms to $1-\mathrm{N}_{2}$ at $69 \mathrm{GPa}$ at $850 \mathrm{~K}$. In view of the relatively high temperature of this transformation and its absence at room temperature, this observation implies that the transformation point is close to the $\theta-1$ equilibrium line (see Fig. 8). At room temperature, $\theta$-nitrogen remains metastable as low as $30 \mathrm{GPa}$ on unloading. Similarly, l-nitrogen remains metastable to $23 \mathrm{GPa}$; at these pressures both phases transform to $\varepsilon-\mathrm{N}_{2}$ on unloading. $\mathrm{l}-\mathrm{N}_{2}$ was found to be stable at low temperatures (down to $10 \mathrm{~K}$ ) at pressures as low as $30 \mathrm{GPa}$. We note that $\eta$ nitrogen so far has been accessed only from $\zeta-\mathrm{N}_{2}$ (see Fig. 8). The apparent kinetic boundary (open circles in Fig. 8) that separates these phases can be treated as a line of instability of $\zeta-\mathrm{N}_{2}$. Likewise, the 1 and $\theta$ phases have been reached only from the $\varepsilon$ phase, though they probably can be formed from $\delta$ as well. We observed that on further increase of pressure and temperature, the $\theta$ phase does not transform to the nonmolecular $\eta$ phase (to at least $135 \mathrm{GPa}$ and $1050 \mathrm{~K}$ ). We suggest that it might instead transform to a (perhaps different) nonmolecular crystalline phase on compression. This nonmolecular phase may not be easily reached by compression at $300 \mathrm{~K}$ because of a kinetic barrier separating it from $\zeta-\mathrm{N}_{2}$.

The above results provide important insights into the behavior of solid nitrogen at high pressures and temperatures. The l-phase appears to represent a different kind of lattice consisting of disk-like molecules, presumably packed more efficiently compared to the mixed disk- and sphere-like $\delta$-family structures. The $\theta$ phase is more complex. Its striking vibrational properties indicate that it is characterized by strong intermolecular interactions, perhaps with some analogy to $\mathrm{H}_{2}$-phase III [51] $\varepsilon-\mathrm{O}_{2}$ [50] or CO2-II [53]. If the interactions are strong enough, the phase may be related to theoretically predicted polyatomic species [54] but this requires further investigation. Our data show that the new phases are thermodynamically stable high-pressure phases since they are formed irrespective of thermodynamic path. Indeed, our data indicate that $\zeta-\mathrm{N}_{2}$ may be metastable in much of the P-T range over which it is observed, since it is typically obtained only as a result of compression at $\mathrm{T}<500 \mathrm{~K}$ of the $\varepsilon-\mathrm{N}_{2}$ (see above). An important general conclusion of this work is that the definitive determination of the equilibrium phase relations of nitrogen is more complex than previously thought due the presence of substantial transformation barriers between different classes of structures. These structures include the well-known phases based on weakly interacting $\mathrm{N}_{2}$ 
16

molecules [10] the recently observed nonmolecular phase and the strongly interacting molecular phases documented here (see also Ref. [48]).

\section{POLYMERIC NITROGEN}

Before this study had began, Raman measurements of nitrogen have been carried out to $130 \mathrm{GPa}$ [24] and $180 \mathrm{GPa}$ [43]. The lowest-frequency vibron has been observed in both studies to the highest pressure reached, and the persistence of this vibron was interpreted as the existence of molecular phase to those pressures. Also, visual observations $[24,43]$ and visible transmission measurements in Ref. [24] reveal color changes at 130-180 GPa but no quantitative characterization has been done.

In contrast to Refs. [24,43], we used only a minute amount of ruby to determine pressure, so the majority of the sample was available for optical measurements. We observe that the Raman and IR vibrons (Fig. 12) lose their intensities in 140-160 GPa pressure range and completely disappear at higher pressures. This is also observed for the Raman and IR lattice modes. It may be argued that the disappearance of the Raman modes is attributed to the presence of a luminescence background (quite moderate with red excitation) and an increase of the visible sample absorption (see below). However, IR intensities are totally independent of these factors because the sample remained transparent in the mid-IR spectral range.
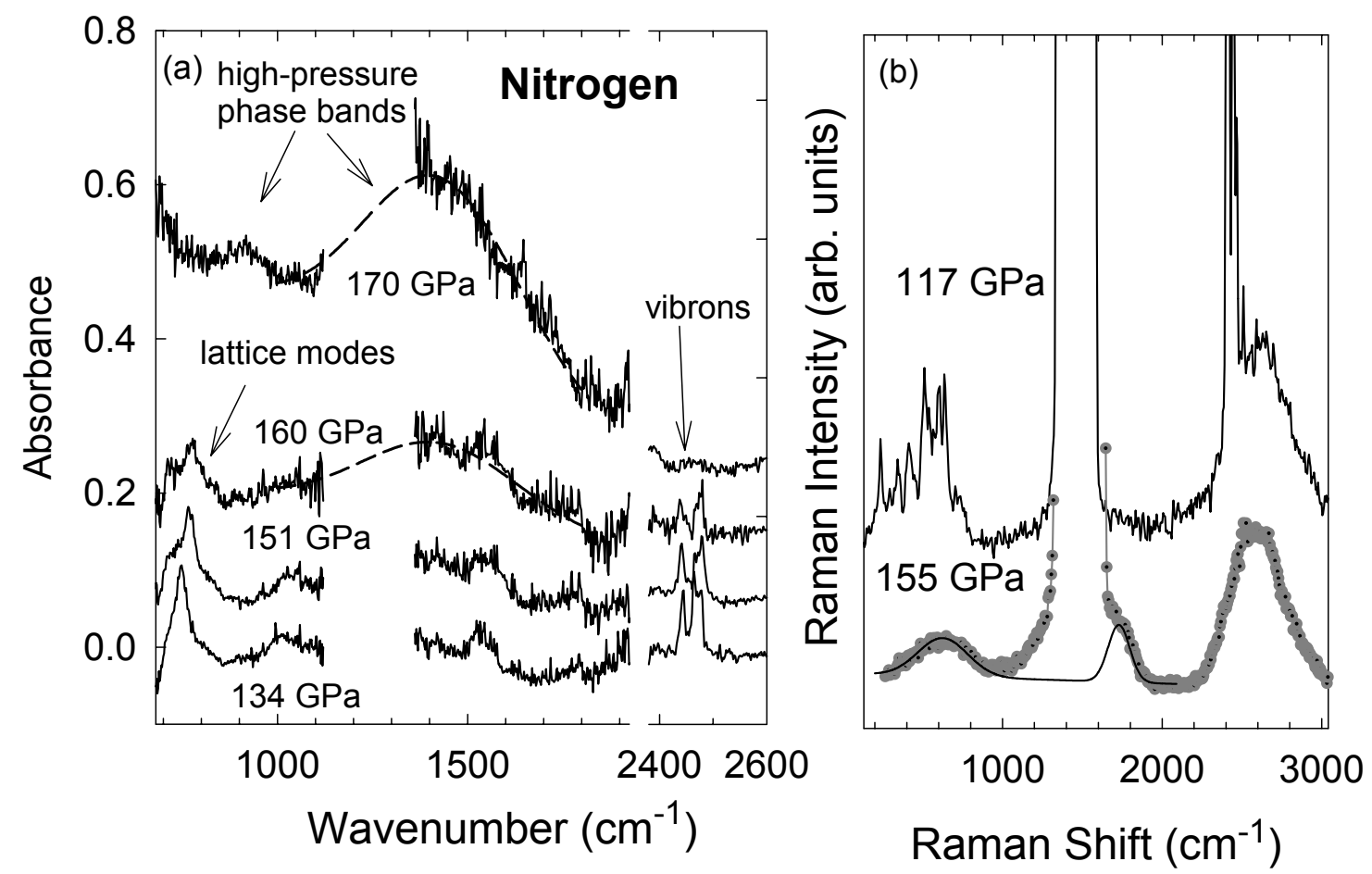

Fig. 12. (a) IR absorption spectra in the vibrational spectral range through the transition to a polymeric phase. Dashed lines are guide to the eye. No data could be obtained in the $1120-1370 \mathrm{~cm}^{-1}$ and $1920-2380$ $\mathrm{cm}^{-1}$ spectral ranges because of strong absorption by the diamonds. (b) Raman spectra through the transition. A strong band at $1350 \mathrm{~cm}^{-1}$ and a broad band at $2600 \mathrm{~cm}^{-1}$ are due to the first and the second order Raman signal of the diamond anvils. The solid line is a guide to the eye. 
Fig. 13 presents the results of visible and IR absorption measurements of nitrogen at elevated pressures. Below $140 \mathrm{GPa}$ nitrogen is transparent in the entire (except the absorption on relatively weak vibrational excitations) measured spectral range (600$20000 \mathrm{~cm}^{-1}$ ). At $150 \mathrm{GPa}$ a wide absorption edge appears in the visible part of the spectra, at which point the sample becomes yellow, and then totally opaque (Fig. 13) at $160 \mathrm{GPa}$. This transformation substantially affected the measurement of IR spectra because of increased absorption in the near-IR range. Nevertheless, we measured IR absorption
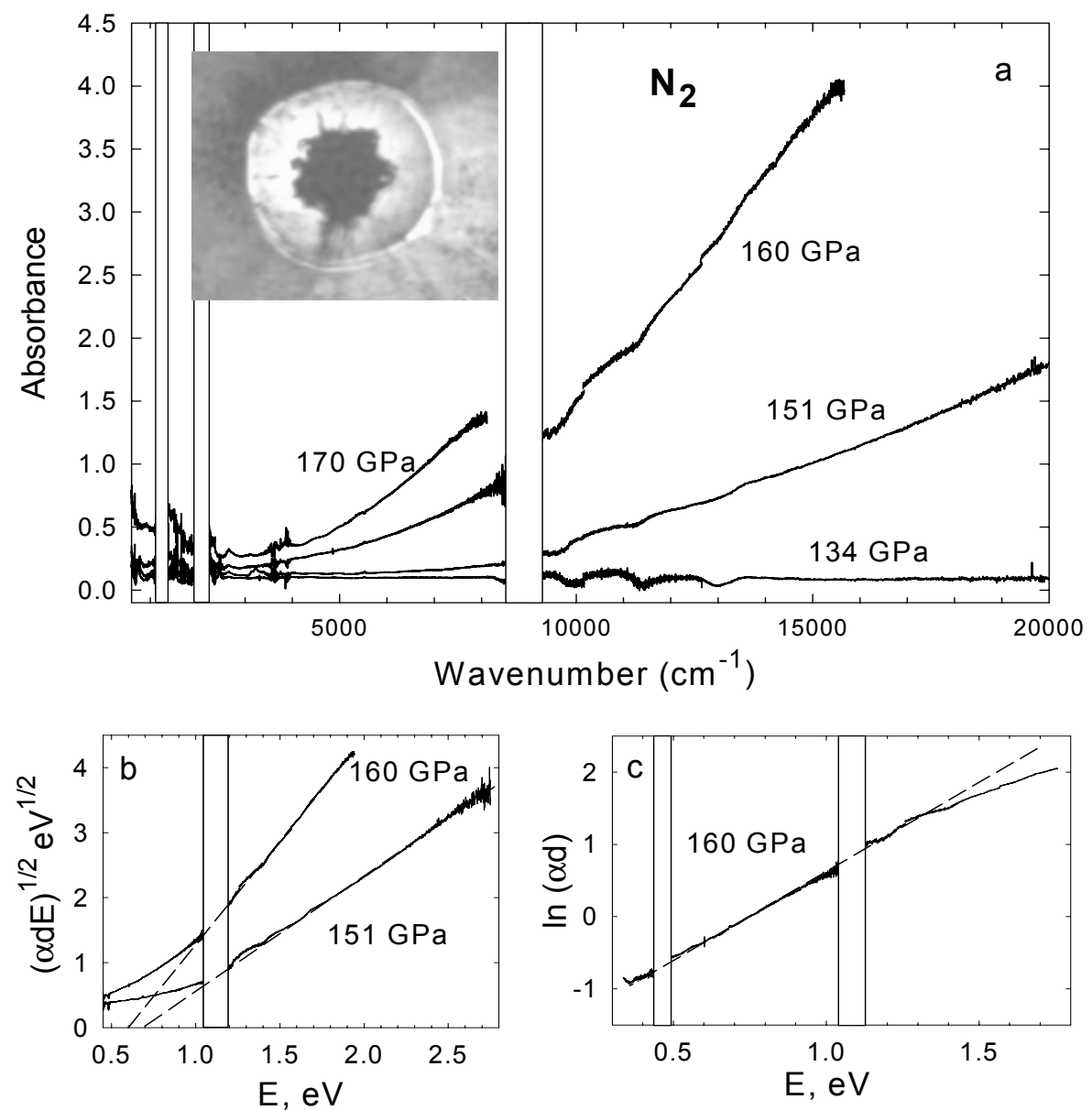

Fig. 13 (a) Optical absorption spectra in a wide spectral range at different pressures (Inset: the microphotography of the opaque sample at $160 \mathrm{GPa}$ (reflected and transmitted light)). (b) Absorption data at 151 and $160 \mathrm{GPa}$ plotted in photon energy $(\mathrm{E})$ versus $(\alpha d \mathrm{E})^{1 / 2}(\alpha$-absorption coefficient, $d$-sample thickness) coordinates illustrating a direct allowed gap absorption law at high energies. Extrapolations of those dependencies to zero absorption give an estimate of the direct gap value. (c) Absorption data at 160 GPa plotted in E versus $\ln (\alpha d)$ coordinates illustrating Urbach-like absorption law at low energies. The frequency ranges of strong diamond absorption are shown by empty boxes. Absorbance $A$ and absorption coefficient $\alpha$ are related by expression $\alpha d=A \ln 10$.

spectra to the highest pressure reached in the experiment (about $170 \mathrm{GPa}$ ). Inspecting the spectra at different pressures above $150 \mathrm{GPa}$ one can easily infer that, to a first 
18

approximation, they can be obtained by simple scaling, indicating an increase of the abundance of the new phase with increasing pressure. This matches closely the vibrational spectroscopic observations of a gradual disappearance of all excitations in the molecular phase (see above) between 140 and $170 \mathrm{GPa}$. The Raman and IR spectra of the new phase show a rather broad, weak Raman band at $640 \mathrm{~cm}^{-1}$ as well as a broader IR band at $1450 \mathrm{~cm}^{-1}$ (Fig. 12). Their intensities seem to increase gradually with pressure, concomitant with a decrease in the intensity of molecular excitations, implying the coexistence of two phases between $140 \mathrm{GPa}$ and $170 \mathrm{GPa}$. The complete change in vibrational excitations and appearance of the low-energy band gap provide evidence for the transformation from the molecular phase to a nonmolecular phase with a narrow gap. Theoretical calculations predict a transformation to threefold-coordinated cubic or distorted cubic structures [3-5] associated with a substantial volume discontinuity (25$33 \%$ ). We can rule out with a high probability the simple cubic high-pressure phase [3], which must be metallic. Other calculations indicated semiconducting phases [3-5], which agree with our experimental data (see also Ref. [48]).

Theoretical calculations $[5,55]$ predict at least a two-fold decrease in the frequency of the $\mathrm{N}-\mathrm{N}$ stretch as a result of the transformation from a triple to a single-bonded molecule. Our IR measurements reveal a broad band in this spectral range (Fig. 12). A Raman band at $640 \mathrm{~cm}^{-1}$ was also observed, which could be identified as a bending mode or correspond to a peak in the phonon density of states (see below). Comparison of our data with the calculations of Barbee (Ref. [55]) shows even more striking qualitative correspondence between experiment and theory assuming the "cubic gauche" [5] highpressure structure (i.e., possible appearance of the second weak IR peak at $900 \mathrm{~cm}^{-1}$ (Fig. 12). A variety of IR and Raman modes are predicted for this phase, and most of them are degenerate. Those modes can split under nonhydrostatic conditions or further lowering of the symmetry Ref. [55], which can explain the observed large linewidth of Raman and IR excitations. Alternatively, the high-pressure phase could be a fine mixture of different tree-coordinate structures with very close total energies e.g. arsenic, black phosphorus, "cubic gauche") and the chain-like structure [5,56] with a high concentration of stacking faults which would make it appear amorphous at the scale of vibrational spectroscopy (see below).

The analysis of the shape of the absorption edge of the high-pressure phase (Fig. 13) shows the existence of two spectral ranges with different types of energy dependence on the absorption coefficient. At high values of absorption it follows the empirical Tauc relation [57] in the case of parabolic band edges (Fig. 13(b)), while at smaller absorption a so-called Urbach or exponential absorption tail [58,59] is observed (Fig. 13(c)). The existence of this kind of absorption edge is normally related to amorphous semiconductors. The optical absorption gap determined from our experiment is 0.6-0.7 $\mathrm{eV}$ and it decreases with pressure (see below). The slope of the Urbach tail, which can be considered as a measure of a random microfield [59] is found to be $\Gamma=2.6 \mathrm{eV}^{-1}$ at 160 $\mathrm{GPa}$. This is very close to what one would expect for an amorphous phase with a coordination of 2.5 [59].

Figure 14 shows representative IR and visible transmission spectra demonstrating the effect of temperature on the semiconducting optical edge characteristic of the $\eta$ phase. The spectra presented correspond to conditions when no molecular phase is present in the sample as determined from IR vibrational spectroscopy. No variation of the shape and position of the band gap can be detected from transmission spectra at different temperatures and constant pressure of $200 \mathrm{GPa}$. Figure 14(b) shows that the low-energy portion of the spectra plotted on a logarithmic scale (Urbach plot) has a constant slope $(\Gamma)$ in a $10-200 \mathrm{~K}$ range. This also agrees with $300 \mathrm{~K}$ data thus showing that $\Gamma$ is not 
temperature dependent. Similar spectra have been reported for amorphous phosphorus at zero pressure [60]. This is typical for solid amorphous semiconductors [59] because the random microfield is caused by static disorder in the system as opposed to crystalline materials [58] where the vibrations generate a temperature-dependent dynamical disorder. Determination of the band gap from our data is a complicated issue, because there is no characteristic feature of the spectra which can be associated with the band gap e.g., Ref. [61]. This is especially important for our measurements, since we essentially deal with samples of various thickness (which is a function of anvil geometry and pressure). As the result, visual observations of the sample above $230 \mathrm{GPa}$ showed that it is red or

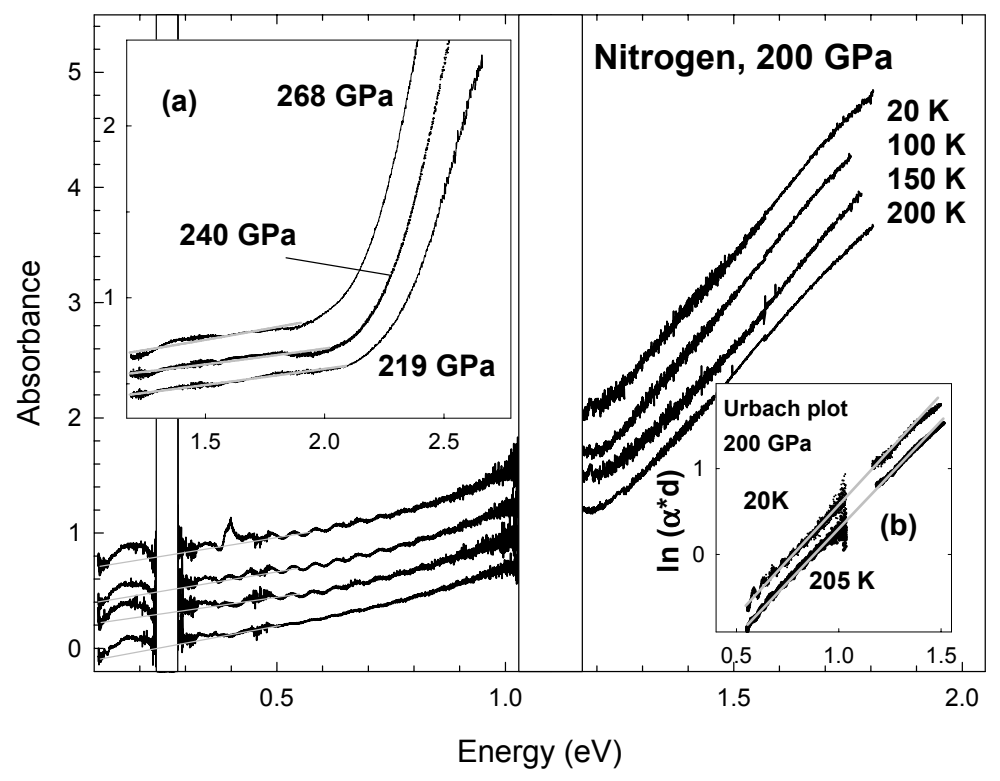

Fig. 14. Transmission spectra of polymeric nitrogen as a function of temperature. Spectra are shifted vertically for clarity. The characteristic peak of the $\eta$ phase is shaded. Inset (a) shows the pressure dependence of the absorption spectra of nitrogen at very high pressures and room temperature. Gray lines represent the Tauc fits to the spectra in an appropriate spectral range. The determination of the energy gap from these measurements is obscured by additional losses caused by the presence of a fine ruby powder in the chamber. The high-energy absorption edge is most probably due to stress-induced absorption of diamond anvils (Ref. 62). (b) Urbach plots at $200 \mathrm{GPa}$ and different temperatures (shifted vertically). Gray lines are guides to the eye.

yellowish in transmission and black in reflection, which is consistent with the semiconducting state. The color of the sample (compare with the observations of dark nitrogen in Refs. 24,37,43,48) may be explained by its thickness of the order of $1 \mu \mathrm{m}$ ) compared to the samples brought to $150 \mathrm{GPa}$ being up to $5 \mu \mathrm{m}$ ). At the highest pressure $(268 \mathrm{GPa})$, visible transmission spectra clearly show the presence of the fundamental absorption edge characteristic of semiconductors (Fig. 14(a)). This result is in agreement with direct electrical measurements performed to $240 \mathrm{GPa}$ [48]. The high-energy 
absorption edge, which can be observed in this case, corresponds to electronic transitions between extended states (unlike Urbach absorption, which is presumably caused by transitions from localized to extended states). Extrapolation of the absorption spectra plotted as $(h \vee \alpha)^{0.5}$ versus $h \vee$ gives the value of optical gap [57]. These values at different pressures are shown in Fig. 15. Note that data from different experiments agree, despite the different sample thickness and the fact that some of the data are taken on pressure release in a metastable pressure region (see below). We observed a monotonic redshift of the band gap with pressure (see also Fig. 14(a)). The pressure dependence of the band gap is sublinear mainly due to contribution from the points obtained on decompression. The extrapolation of the band gap values gives metallization at pressures slightly above $300 \mathrm{GPa}$. Linear extrapolation of this curve to higher pressures (not taking into account points obtained upon decompression) gives a value of $280 \mathrm{GPa}$.

We now present temperature measurements of the vibrational properties of the $\eta$ phase. Type II diamonds were used for mid-IR measurements to avoid interference with the characteristic absorption of the sample. The representative absorption spectra at different temperatures (see Fig. 14) clearly show the presence of a broad $1700 \mathrm{~cm}^{-1}$ IR band (compare with Fig. 12). Its presence was also observed in the sample heated to $495 \mathrm{~K}$ at $117 \mathrm{GPa}$ (see below). The position of the band and its damping (if fitted as one band) does not depend on pressure and temperature within the error bars. The Raman spectrum of the $\eta$ phase obtained on heating (see below) does not show any trace of the molecular phase (see Fig. 12(b)). Careful examination of the spectrum in this case showed a weak broad band at $640 \mathrm{~cm}^{-1}$ and a shoulder near $1750 \mathrm{~cm}^{-1}$ (both indicated by arrows in Fig. 12(b)). For an amorphous state, the vibrational spectrum would closely resemble a density of phonon states [63] with the maxima corresponding roughly to the zone boundary acoustic and optic vibrations of an underlying structure [3-5,55], which is
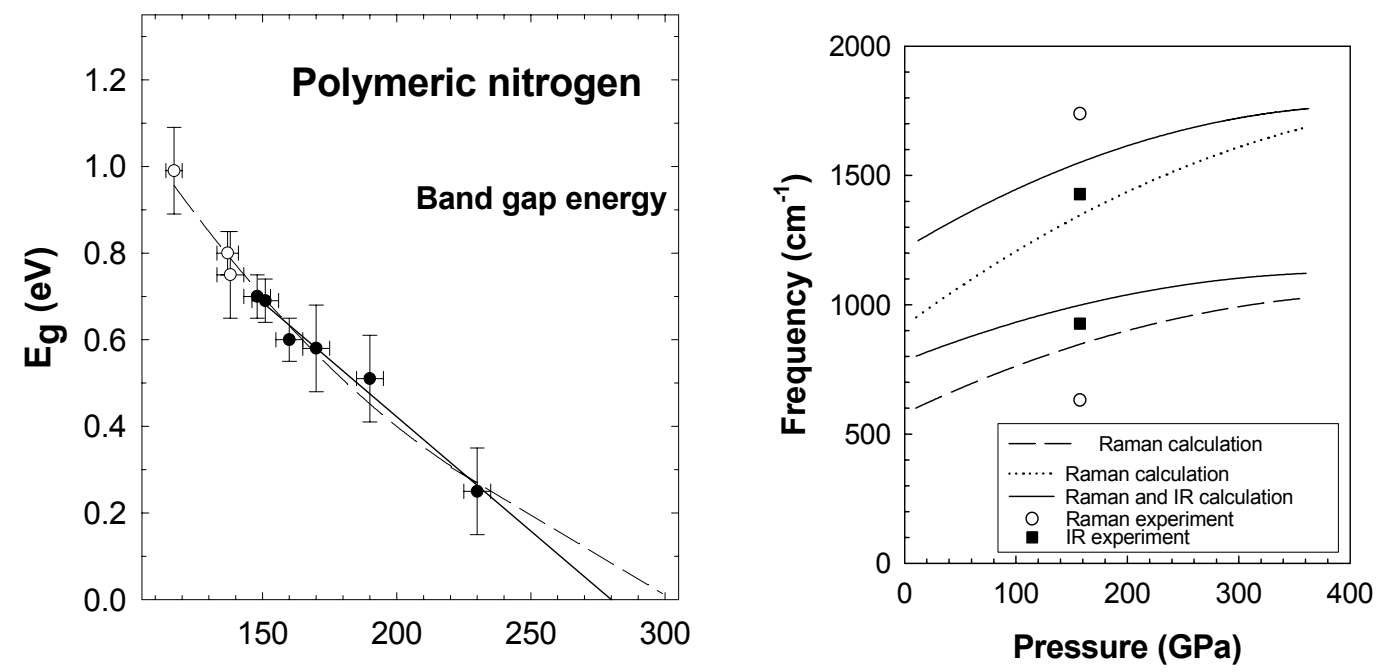

Fig. 15. Band gap of the $\eta$ phase as a function of pressure. Solid circles represent increasing pressure and open circles -decreasing pressure. Linear and quadratic extrapolations are shown as dashed lines.

Fig. 16. Comparison of the measured (symbols) and calculated (lines) vibrational frequencies for the cubic gauche structure [55].

consistent with our observations. The only lattice dynamics calculations for hypothetical high-pressure crystalline phases of nitrogen are available for the cubic gauche phase [55] 
and calculated phonon frequencies are in a qualitative agreement with our measurements (Fig. 16). The vibrational spectroscopy and band gap structure indicate the absence of a long-range order. The material can still possess some short-range order, for example, related to pyramidal coordination of nitrogen atoms. The absence of long-range order can be due to structural flexibility because each atom forms bonds with only three other atoms out of six nearest neighbors [3-5].

We probed the forward and reverse transformations of the molecular to the $\eta$ phase in different regions of $P-T$ space (Fig. 8) We used IR transmission spectra as diagnostics of the degree of transformation to the nonmolecular phase. The absence of IR bands corresponding to vibrons and lattice modes of the molecular phase was used as a criterion. Since both the molecular and nonmolecular phases are transparent in the midIR, the amount of the phase present is simply proportional to the amplitude of the corresponding IR peaks. This is unlike the situation with Raman spectra, which are attenuated by absorption of the $\eta$ phase. We examined the transformation at $205 \mathrm{~K}$ and elevated pressures and found that it starts at $155 \mathrm{GPa}$ and is completed at $185 \mathrm{GPa}$. This is shifted to higher pressures compared to our $300 \mathrm{~K}$ data and is in agreement with the trend reported in Ref. [48]. The sample has been cooled down to $10 \mathrm{~K}$ at $200 \mathrm{GPa}$ and warmed up after subsequent release of pressure at 130-150 GPa. IR and visible transmission spectra and Raman spectra clearly showed the persistence of the $\eta$ phase without any reverse transformation down to $120 \mathrm{GPa}$. At this point the pressure dropped to $87 \mathrm{GPa}$ and the sample transformed instantaneously back to a transparent phase (called $\zeta$ 'here). The molecular nature of this phase is confirmed by its Raman spectrum although the positions of the vibron lines do not correspond to those observed on pressure increase [38]. This means that the amorphous phase back-transforms to a molecular phase, which differs from the one observed on upstroke. On further release of pressure (to $60 \mathrm{GPa}$ ) we observe the Raman spectra, which are similar to those of $\varepsilon$ phase in positions and intensities of vibron peaks.

In the heating experiment we first exposed the sample to $495 \mathrm{~K}$ at $117 \mathrm{GPa}$. The effect of temperature caused a gradual transformation (starting at $450 \mathrm{~K}$ ) similar to that observed at 300 and $200 \mathrm{~K}$. A large increase of fluorescence typically precedes transformation to the $\eta$ phase. Once the transformation is completed (see below), this fluorescence disappears (Fig. 12(b)). The comparison of Raman modes revealed more than a tenfold decrease of intensity in the Raman vibrons and no observable lattice modes. Quenching of the sample to room temperature did not change the color and visible absorption spectra. Surprisingly, the infrared spectra revealed the presence of molecular vibrons, indicating an incomplete transformation (about 30\% of the nonmolecular phase judging from the infrared activity). During the second heating the sample was completely transformed to the $\eta$ phase. Then the pressure was dropped to $105 \mathrm{GPa}$ at $460 \mathrm{~K}$, causing an instantaneous reverse transformation to a transparent molecular phase. The spectral positions of the bands and their number do not correspond to those observed at this pressure on compression but are similar to those obtained during the unloading at $300 \mathrm{~K}$ (see above). Increasing pressure to $135 \mathrm{GPa}$ at $510 \mathrm{~K}$ drove the direct transformation into the $\eta$ phase again. Figure 8 summarizes our data for the phase diagram of nitrogen obtained in a course of extensive $P-T$ measurements. Substantial hysteresis is observed for the transformation from and back to the molecular phase, so the observed curves should be treated as kinetic boundaries. For a direct transformation, our data are in good agreement with the results of visual observations of Ref. 48. Our high-temperature data show that the hysteresis becomes quite small at temperatures above $500 \mathrm{~K}$. There is large hysteresis at lower temperature such that the molecular $\zeta$ phase can be metastably retained beyond the $\zeta-\zeta$ ' boundary (above approximately $100 \mathrm{GPa}$; see also Ref. 48). Thus, observation of another molecular phase ( $\zeta$ ') in this $P-T$ conditions means that this 
22

phase is either kinetically favored or thermodynamically stable with respect to the $\zeta$ phase.

If the potential barrier between two crystalline phases is high (molecular dissociation is required in our case), the transition may be preempted by a transformation to a metastable phase, which may be amorphous [64]. This defines an intrinsic stability limit (e.g., spinodal) for the diatomic molecular state of nitrogen. In view of the amorphous component of the higher-pressure phase, the transition may be considered as a type of pressure-induced amorphization. As such, the transformation boundary could track the metastable extension of the melting line of the molecular phase, and if so, it should have a negative slope (consistent with negative $\Delta V$ and positive $\Delta S$ for a transition to dense amorphous state [64]. Alternatively, one can view this in terms of an intrinsic (elastic or dynamical) instability of the structure of the molecular solid. In this sense, the behavior of the material parallels other amorphizing systems that undergo coordination changes (see Ref. [65]).

In conclusion, we present optical evidence for a transition of molecular nitrogen to a nonmolecular state. The transition occurs on compression when the ratio of inter-tointramolecular force constants reaches 0.1 [37]. This is small compared to the highest ratio reached for hydrogen (in its molecular phase). It suggests that the destabilization of the triple-bonded nitrogen molecule is the driving force of the nonmolecular transition. Vibrational and optical spectroscopic data indicate that the high-pressure phase is a narrow-gap, disordered, and single-bonded phase. The amorphous nature of the highpressure phase may represent the common case of a transition in a field of deep metastability [66].

\section{Acknowledgement}

This work was supported by the Carnegie/DOE Alliance Center (CDAC), which is supported by the DOE/NNSA, and by NSF-DMR, NASA, and the W. M. Keck Foundation. Work at Lawrence Livermore National Laboratory was performed under the auspices of the University of California under DOE Contract No. W-7405-Eng-48.

\section{Bibliography}

[1] R. J. Hemley and N. W. Ashcroft, Phys. Today, 51(8), 26 (1998).

[2] H. B. Radousky et al., Phys. Rev. Lett. 57, 2419 (1986).

[3] A. K. McMahan and R. LeSar, Phys. Rev. Lett. 54, 1929 (1985).

[4] R. M. Martin and R. J. Needs, Phys. Rev. B 34, 5082 (1986).

[5] C. Mailhiot, L. H. Yang, and A. K. McMahan, Phys. Rev. B 46, 14419 (1992).

[6] J. Belak, R. LeSar, and R. D. Etters, J. Chem. Phys. 92, 5430 (1990)

[7] S. Nosé and M. L. Klein, Phys. Rev. Lett. 50, 1207 (1983)

[8] R. D. Etters, V. Chandrasekharan, E. Uzan, and K. Kobashi, Phys. Rev. B 33, 8615 (1986)

[9] R. Bini, L. Ulivi, J. Kreutz, and H. Jodl, J. Chem. Phys. 112, 8522 (2000).

[10] V. G. Manzhelii, Y. A. Freiman, Physics of cryocrystals, American Institute of Physics, College Park MD, 1997

[11] D. A. Young et al., Phys. Rev. B 35, 5353 (1987).

[12] S. Zinn, D. Schiferl, and M. F. Nicol, J. Chem. Phys. 87, 1267 (1987)

[13] W. L. Vos and J. A. Schouten, J. Chem. Phys. 91, 6302 (1989). 
[14] S. C. Schmidt, D. Schiferl, A. S. Zinn, D. D. Ragan, and D. S. Moore J. Appl. Phys. 69 (5), 2793 (1991).

[15] C. A. Swenson, J. Chem. Phys. 23, 1963 (1955).

[16] R. L. Mills and A. F. Schuch, Phys. Rev. Lett. 23, 1154 (1969)

[17] F. Schuch and R. L. Mills, J. Chem. Phys. 52, 6000 (1970)

[18] J. R. Brookeman and T. A. Scott, J. Low. Temp. Phys. 12, 491 (1973)

[19] W. E. Streib, T. H. Jordan, and W. N. Lipscomb, J. Chem. Phys. 37,2962 (1962)

[20] R. LeSar, S. A. Ekberg, L. H. Jones, R. L. Mills, L. A. Schwalbe, and D. Schiferl, Solid State Comm. 32, 131 (1979)

[21] S. Buchsbaum, R. L. Mills, and D. Schiferl, J. Phys. Chem. 88, 2522 (1984)

[22] D. T. Cromer, R. L. Mills, D. Schiferl, and L. A. Schwalbe, Acta Crystallogr. B 37, $8(1981)$

[23] D. Schiferl, S. Buchsbaum, and R. L. Mills, J. Phys. Chem. 89, 2324 (1985)

[24] R. Reichlin, D. Schiferl, S. Martin, C. Vanderborgh, and R. L. Mills, Phys. Rev.

Lett. 55, 1464 (1985)

[25] R. L. Mills, B. Olinger, and D. T. Cromer, J. Chem. Phys. 84, 2837 (1986)

[26] H. Olijnyk, J. Chem. Phys. 93, 8968 (1990)

[27] H. Schneider, W. Haefner, A. Wokaun, and H. Olijnyk, J. Chem. Phys. 96, 8046 (1992)

[28] M. I. M. Scheerboom and J. A. Schouten, Phys. Rev. Lett. 71, 2252 (1993)

[29] M. I. M. Scheerboom and J. A. Schouten, J. Chem. Phys. 105, 2553 (1996)

[30] R. Bini, M. Jordan, L. Ulivi, and H. J. Jodl, J. Chem. Phys. 108, 6849 (1998)

[31] A. Mulder, J. P. J. Michels, and J. A. Schouten, J. Chem. Phys. 105, 3235 (1996)

[32] A. Mulder, J. P. J. Michels, and J. A. Schouten, Phys. Rev. B 57, 7571 (1998)

[33] M. Hanfland, M. Lorenzen, C. Wassilew-Reul, and F. Zontone, in Abstracts of the

International Conference on High Pressure Science and Technology, Kyoto, Japan, 1997,

p. 130

[34] T. Westerhoff, A. Wittig, and R. Feile, Phys. Rev. B. 54, 14 (1996)

[35] H. Olijnyk and A. P. Jephcoat, Phys. Rev. Lett. 83, 332 (1999)

[36] A. P. Jephcoat, R. J. Hemley, H. K. Mao, and D. E. Cox, Bull. Am. Phys. Soc. 33, $522(1988)$

[37] A. F. Goncharov, E. Gregoryanz, H.-k. Mao, Z. Liu, and R. J. Hemley. Phys. Rev.

Lett., 2000, 85, 1262-1265.

[38] E. Gregoryanz, A. F. Goncharov, R. J. Hemley, and H-k. Mao. Phys. Rev. B 64, 052103 (2001).

[39] A. F. Goncharov, E.. Gregoryanz, H-k. Mao, and R.J. Hemley. Fizika Nizkikh Temperatur, 27, 1170-1174 (2001).

[40] E. Gregoryanz, A. F. Goncharov, R. J. Hemley, H-K. Mao, M. Somayazulu, and G. Shen, , Phys. Rev. B 66, 224108 (2002).

[41] A. F. Goncharov, V. V. Struzhkin, R. J. Hemley, H. K. Mao, and Z. Liu, in:

Science and Technology of High Pressure, edited by M. H. Manghnani, W. J. Nellis and M. F. Nicol (Universities Press, Hyderabad, India, Honolulu, Hawaii, 1999), Vol. 1, p. 90-95.

[42] Y. Fei, in Mineral Spectroscopy: A Tribute to Roger G. Burns, edited by M.D.

Dyar, C. McCammon and M. W. Schafer (Geochemical Society, Houston, 1966) p. 243.

[43] P. M. Bell, H. K. Mao, R. J. Hemley, Physica 139\\&140B, 16 (1986).

[44] M. D. McCluskey, L. Hsu, L. Wang, and E. E. Haller, Phys. Rev. B \{lbf 54\}, 8962 (1996).

[45] R. Bini, M. Jordan, L. Ulivi, H. J. Jodl, J. Chem. Phys. 106, 6849 (1998).

[46] D. Hohl, V. Natoli, D. M. Ceperley, and R. M. Martin, Phys. Rev. Lett. 71, 541 (1993).

[47] R. Jeanloz, J. Geophys. Res. 92, 10352 (1987)

[48] M. Eremets, R. J. Hemley, H. K. Mao, and E. Gregoryanz, Nature 
(London) 411, 170 (2001).

[49] M. Hanfland, R. J. Hemley, and H. K. Mao, Phys. Rev. Lett. 70, 3760 (1993).

[50] F. Gorelli, L. Ulivi, M. Santoro, and B. Bini, Phys. Rev. Lett. 83, 4093 (1999).

[51] J. Hemley, Z. Soos, M. Hanfland, and H. K. Mao, Nature (London) 369, 384 (1994).

[52] J. Kohanoff, S. Scandolo, S. Gironcoli, and E. Tosatti, Phys. Rev. Lett. 83, 4097

(1999); I. I. Mazin, R. J. Hemley, A. F. Goncharov, M. Hanfland, and H. K. Mao, ibid. 78, 1066 (1997).

[53] Iota and C. S. Yoo, Phys. Rev. Lett. 86, 5922 (2001).

[54] R. Bartlett, Chem. Ind. 4, 140 (2000).

[55] T. W. Barbee III, Phys. Rev. B. 48, 9327 (1993).

[56] M. M. G. Alemany, J. L. Martins, Phys. Rev. B 68, 024110 (2003).

[57] J. Tauc, R. Grigorovici, and A. Vancu, Phys. Status Solidi, 15, 627 (1966).

[58] F. Urbach, Phys Rev. 92, 1324 (1953).

[59] N. F. Mott and E. A. Davis, Electronic Processes in Non-Crystalline Materials, 2nd

Ed., Clarendon Press, Oxford (1979).

[60] L. J. Pilione, R. J. Pomian, and J. S. Lannin, Solid State Commun. 39, 933 (1981).

[61] S. Knief, Phys. Rev. B 59, 12940 (1999).

[62] Y. K. Vohra, in Recent Trends in High Pressure Research, edited by A. K. Singh (Oxford \& IBH, Calcutta, 1991), p. 349.

[63] M.H. Brodski in: Light scattering in solids, Topics in Applied Physics, vol. 8, edited by M. Cardona (Springer-Verlag, New York 1983).

[64] For recent reviews see S. M. Sharma and S. K. Sikka, Prog. Mat. Sci. 40, 1 (1996); P. Richet and P. Gillet, Eur. J. Mineral. 9, 907 (1997).

[65] R. J. Hemley, A. Jephcoat, H. Mao, L. Ming, and M. Manghnani, Nature (London) 334, 52 (1988); J. Badro, J.-L. Barrat, and P. Gillet, Europhys. Lett. 42, 643 (1998). This includes the likelihood that material produced on compression is heterogeneous (i.e., partly crystalline); see R. J. Hemley, J. Badro, and D. M. Teter, in Physics Meets Mineralogy, edited by H. Aoki, Y. Syomo, and R. Hemley (Cambridge University Press, Cambridge, England, 2000), p. 173.

[66] E. G. Ponyatovsky and O. I. Barkalov, Mater. Sci. Rep. 8, 1471 (1992).

(the one starting at the left with USER GUIDELINES).

If it is not then go to 'View' ( 'Toolbars' and tick 'Elsevier'.

Please have macros enabled when loading

to use the functionality of this template.

(Go to 'Tools' ( 'Macro' ( 'Security' and choose 'Medium') 